\title{
Adaptive Stabilization for Nonholonomic Systems with Unknown Time Delays
}

\author{
Yuanyuan Wu, ${ }^{1,2}$ Qingbo $\mathrm{Li}^{3}{ }^{3}$ and Yuqiang $\mathrm{Wu}^{4}$ \\ ${ }^{1}$ College of Electric and Information Engineering, Zhengzhou University of Light Industry, Zhengzhou, Henan 450002, China \\ ${ }^{2}$ Department of Mathematics, Southeast University, Nanjing, Jiangsu 210096, China \\ ${ }^{3}$ Department of Mathematics and Information Science, Zhengzhou University of Light Industry, Zhengzhou, Henan 450002, China \\ ${ }^{4}$ Research Institute of Automation, Qufu Normal University, Qufu, Shandong 273165, China
}

Correspondence should be addressed to Yuanyuan Wu; yuanyuan539@yahoo.com.cn

Received 19 June 2013; Revised 20 October 2013; Accepted 3 November 2013

Academic Editor: Oleg V. Gendelman

Copyright (C) 2013 Yuanyuan Wu et al. This is an open access article distributed under the Creative Commons Attribution License, which permits unrestricted use, distribution, and reproduction in any medium, provided the original work is properly cited.

\begin{abstract}
This paper presents an adaptive control strategy for a class of nonholonomic systems in chained form with virtual control coefficients, nonlinear uncertainties, and unknown time delays. State scaling technique and backstepping recursive approach are applied to design a nonlinear state feedback controller, which can guarantee the stabilization of the closed-loop systems. The simulation results are provided to show the effectiveness of the proposed method.
\end{abstract}

\section{Introduction}

In the last few decades, considerable efforts have been devoted to the research of nonholonomic system, which is a particular class of nonlinear systems and widespread in real world, such as mobile robots, car-like vehicle, underactuated satellites, and knife-edge. It is well known that the control of nonholonomic systems is extremely challenging, largely due to the impossibility of asymptotically stabilizing nonholonomic systems via smooth time-invariant state feedback, a well-recognized fact pointed out in $[1,2]$. In order to overcome this obstruction, several approaches have been proposed, such as discontinuous feedback, time-varying feedback, and hybrid stabilization.

The discontinuous feedback stabilization was first proposed in [3], and then further discussion was made in [47]; especially an elegant discontinuous coordinate transformation approach was presented in [5] for the stabilization problem of nonholonomic systems. Meanwhile, the smooth time-varying feedback control strategies also have drawn much attention [8-11]. To date, there have been several controller design approaches for the asymptotic stabilization or exponential regulation of nonholonomic control systems [4-14].
As pointed out in [9], many nonlinear mechanical systems with nonholonomic constraints can be transformed, either locally or globally, into the nonholonomic systems in the so-called chained form. Therefore, a number of research literature resources [8-23] for such chained nonholonomic systems are provided. Recently, some new adaptive control strategies have been proposed to stabilize the nonholonomic systems. For instance, state feedback control is studied in [15$20]$ and output feedback control in [21-25].

From a practical point of view, when modeling a mechanical system, time delay should be taken into account, and there are a few literature resources $[19,20,25]$ for the nonholonomic systems with time delay. In $[19,20,25]$, the problem of stabilization is studied for delayed nonholonomic systems; however, the virtual control coefficients and unknown parameter vector are not considered.

In this paper, we introduce a new class of chained nonholonomic systems with unknown virtual control coefficients, uncertain nonlinearities, and unknown time delays and then study the problem of adaptive state feedback stabilization. Since the nonholonomic system considered in this paper contains the delayed terms, it cannot be handled by existing conventional methods. The proposed constructive design method is based on a combined application of the state 
scaling technique, the recursive backstepping approach, and the novel Lyapunov-Krasovskii functionals. The switching control strategy for the first subsystem is employed to achieve the asymptotic stabilization.

\section{Problem Formulation and Preliminaries}

In this paper, we present an adaptive stabilization control design procedure for the following nonholonomic systems with nonlinear uncertainties and unknown time delays:

$$
\begin{gathered}
\dot{x}_{0}(t)=d_{0} u_{0}(t)+\phi_{0}\left(t, x_{0}(t), \theta\right), \\
\dot{x}_{1}(t)=d_{1} u_{0}(t) x_{2}(t)+\varphi_{1}\left(y(t), y\left(t-\tau_{1}\right)\right) \\
+\phi_{1}\left(t, u_{0}(t), x_{0}(t), x(t), \theta\right), \\
\vdots \\
\dot{x}_{i}(t)=d_{i} u_{0}(t) x_{i+1}(t)+\varphi_{i}\left(y(t), y\left(t-\tau_{i}\right)\right) \\
+\phi_{i}\left(t, u_{0}(t), x_{0}(t), x(t), \theta\right), \\
\vdots \quad \dot{x}_{n}(t)=d_{n} u_{1}(t)+\varphi_{n}\left(y(t), y\left(t-\tau_{n}\right)\right) \\
\quad+\phi_{n}\left(t, u_{0}(t), x_{0}(t), x(t), \theta\right), \\
y(t)=\left[x_{0}(t), x_{1}(t)\right]^{T},
\end{gathered}
$$

where $\left[x_{0}(t), x(t)\right]^{T}=\left[x_{0}(t), x_{1}(t), \ldots, x_{n}(t)\right]^{T} \in R^{n+1}$ and $u(t)=\left[u_{0}(t), u_{1}(t)\right]^{T} \in R^{2}$ are system states and control input, respectively. $\theta \in R^{m}$ is an unknown bounded parameter vector. $d_{i}(0 \leq i \leq n)$ are disturbed virtual control coefficients, and the individual signs are known. $\varphi_{i}^{d}\left(y(t), y\left(t-\tau_{i}\right)\right)^{\prime} s$ denote the delayed terms, which contain output delays. $\tau_{i}(1 \leq i \leq n)$ are unknown constants; $\phi_{i} \in$ $R^{m}$ are vectors of smooth nonlinear functions and represent unmodeled dynamic and external disturbances.

Assumption 1. For nonlinear functions $\phi_{0}$ and $\phi_{i}(1 \leq i \leq n)$, there exist (known) smooth nonnegative functions $\bar{\phi}_{0}$ and $\bar{\phi}_{i j}(1 \leq j \leq i \leq n)$ such that

$$
\begin{gathered}
\phi_{0}\left(t, x_{0}(t), \theta\right)=x_{0} \bar{\phi}_{0}\left(x_{0}, \theta\right), \\
\left|\phi_{i}\left(t, u_{0}, x_{0}, x_{i}, \theta\right)\right| \leq\left|x_{0}\right|^{n-i+1} \bar{\phi}_{i 0}\left(x_{0}, x_{1}, \theta\right) \\
+\sum_{j=1}^{i}\left|x_{j}\right| \bar{\phi}_{i j}\left(u_{0}, x_{0}, \bar{x}_{i}, \theta\right),
\end{gathered}
$$

where $\bar{x}_{i}=\left[x_{1}, \cdot, x_{i}\right]^{T}$.

Assumption 2. The nonlinear functions $\varphi_{i}\left(y(t), y\left(t-\tau_{i}\right)\right)(1 \leq$ $i \leq n)$ satisfy

$$
\begin{aligned}
& \left|\varphi_{i}\left(y(t), y\left(t-\tau_{i}\right)\right)\right| \\
& \quad \leq\left|x_{1}(t) x_{1}\left(t-\tau_{i}\right)\right| \bar{\varphi}_{i}\left(y(t), y\left(t-\tau_{i}\right)\right),
\end{aligned}
$$

in which $\bar{\varphi}_{i}$ are (known) smooth nonnegative nonlinear functions.

Remark 3. It is clear that system (1) covers a number of important classes of uncertain nonholonomic systems that have been investigated in some existing literature resources. For instance, when $d_{i}=1$ and $\phi_{i}=0, \phi_{i=0}$ system (1) reduces the standard form of nonholonomic system which has been widely studied in the literature [15, 18-20]. Moreover, in Ge et al. [15], not only the virtual control coefficients $d_{i}=1$ and the dynamics $\phi_{i}$ satisfying $\phi_{i}=\widetilde{\phi}_{i}^{T} \theta$ are assumed but also the modeled dynamics $\varphi_{i}$ do not exist. In Liu and Zhang [22], the virtual control coefficients and time delays have not been considered, and the expression $\phi_{i}=\widetilde{\phi}_{i}^{T} \theta$ is also required. While $d_{i}=1$ and $\varphi_{i}$ and unknown parameters $\theta$ are not existent, system (1) degenerates to the one studied in $\mathrm{Xi}$ et al. [21]. When $\varphi_{i}=0$, together with $\phi_{i}=\widetilde{\phi}_{i}^{T} \theta$, system (1) becomes the considered system in Ju et al. [23].

Remark 4 . Note that here we only use the sign of $d_{i}$ without any knowledge of individual virtual control coefficient $d_{i}(1 \leq$ $i \leq n)$. Moreover, Assumptions 1 and 2 are imposed on the nonlinear functions $\phi_{i}$ and the delayed terms $\varphi_{i}$ of system (1), respectively. It can be seen that some similar conditions are implied in [22].

Lemma 5. For any real-valued continuous function $f(x, y)$, where $x \in R^{n}$ and $y \in R^{m}$, there are smooth functions $a(x) \geq 0$, $b(y) \geq 0, c(x) \geq 1$ and $d(y) \geq 1$ such that

$$
|f(x, y)| \leq a(x)+b(y), \quad|f(x, y)| \leq c(x) d(y) .
$$

By Lemma 5 and Assumption 1, we know that there exist smooth functions $\omega_{0} \geq 1, \omega_{i 0} \geq 1, \omega_{i j} \geq 1, \zeta_{0} \geq 1, \zeta_{i 0} \geq 1$, and $\zeta_{i j} \geq 1$ such that

$$
\begin{gathered}
\left|\phi_{0}\left(t, x_{0}(t), \theta\right)\right| \leq\left|x_{0}\right| \omega_{0}\left(x_{0}\right) \zeta_{0}(\theta) \\
\left|\phi_{i}\left(t, u_{0}, x_{0}, x_{i}, \theta\right)\right| \leq\left|x_{0}\right|^{n-i+1} \omega_{i 0}\left(x_{0}, x_{1}\right) \zeta_{i 0}(\theta) \\
+\sum_{j=1}^{i}\left|x_{j}\right| \omega_{i j}\left(u_{0}, x_{0}, \bar{x}_{i}\right) \zeta_{i j}(\theta) .
\end{gathered}
$$

Denote that $\vartheta=\zeta_{0}+\sum_{i=1}^{n} \sum_{j=0}^{i} \zeta_{i j}(\theta)$, then the above inequalities can be rewritten as follows:

$$
\begin{gathered}
\left|\phi_{0}\left(t, x_{0}(t), \theta\right)\right| \leq\left|x_{0}\right| \omega_{0}\left(x_{0}\right) \vartheta, \\
\left|\phi_{i}\left(t, u_{0}, x_{0}, x_{i}, \theta\right)\right| \leq\left|x_{0}\right|^{n-i+1} \omega_{i 0}\left(x_{0}, x_{1}\right) \vartheta \\
+\sum_{j=1}^{i}\left|x_{j}\right| \omega_{i j}\left(u_{0}, x_{0}, \bar{x}_{i}\right) \vartheta .
\end{gathered}
$$

\section{Adaptive Stabilization Control Design}

In this section, we will design an adaptive stabilization controller for the case that $x_{0}(t) \neq 0$, and the case that $x_{0}(t)=$ 0 will be considered in the next section. Now, we use two 
separate stages to globally asymptotically stabilize the system (1). Firstly, the control $u_{0}(t)$ should be designed for $x_{0^{-}}$ subsystem; in the second stage, we design $u_{1}(t)$ to guarantee all states of the rest in system (1) converge to zero.

3.1. State Scaling. The following state scaling discontinuous transformation is introduced:

$$
z_{i}(t)=\frac{x_{i}(t)}{x_{0}^{n-i}(t)}, \quad 1 \leq i \leq n
$$

Under the new $z$-coordinates, the system (1) is transformed into

$$
\begin{aligned}
& \dot{x}_{0}(t)=d_{0} u_{0}(t)+\phi_{0}\left(t, x_{0}(t), \theta\right) \\
& \dot{z}_{i}(t)=\frac{u_{0}(t)}{x_{0}(t)} d_{i} z_{i+1}(t)-(n-i) \frac{u_{0}(t)}{x_{0}(t)} d_{0} z_{i}(t)+\widetilde{\phi}_{i}+\widetilde{\varphi}_{i}, \\
& \dot{z}_{n}(t)=d_{n} u_{1}(t)+\widetilde{\phi}_{n}+\widetilde{\varphi}_{n},
\end{aligned}
$$

where

$$
\begin{aligned}
\tilde{\phi}_{i}= & \frac{\phi_{i}^{d}\left(t, u_{0}(t), x_{0}(t), x(t), \theta\right)}{x_{0}^{n-i}(t)} \\
& -\frac{(n-i) \phi_{0}\left(t, u_{0}(t), x_{0}(t), x(t), \theta\right)}{x_{0}(t)} z_{i}(t), \\
\widetilde{\varphi}_{i}= & \frac{\varphi_{i}\left(y(t), y\left(t-\tau_{i}\right)\right)}{x_{0}^{n-i}(t)} .
\end{aligned}
$$

In order to obtain the estimation for the nonlinear functions $\widetilde{\phi}_{i}$ and $\widetilde{\varphi}_{i}$, the following lemmas are given.

Lemma 6. For every $1 \leq i \leq n$, there exist smooth nonnegative functions $\widetilde{\omega}_{i 0}\left(x_{0}, z_{1}\right)$ and $\widetilde{\omega}_{i j}\left(u_{0}, x_{0}, \bar{z}_{i}\right)$ such that

$$
\begin{aligned}
\left|\widetilde{\phi}_{i}\right| \leq & \left|x_{0}(t)\right| \widetilde{\omega}_{i 0}\left(x_{0}(t), z_{1}(t)\right) \vartheta \\
& +\sum_{j=1}^{i}\left|z_{j}(t)\right| \widetilde{\omega}_{i j}\left(u_{0}(t), x_{0}(t), \bar{z}_{i}(t)\right) \vartheta
\end{aligned}
$$

where $\bar{z}_{i}=\left[z_{1}, \ldots, z_{i}\right]^{T}$.
Proof. By the above inequalities (6), it can be deduced that

$$
\begin{aligned}
\left|\tilde{\phi}_{i}\right| \leq & \frac{\left|\phi_{i}\left(t, u_{o}, x_{0}, x, \theta\right)\right|}{\left|x_{0}^{n-i}\right|}+\frac{\left|(n-i) \phi_{0}\left(t, x_{0}, \theta\right)\right|}{\left|x_{0}\right|}\left|z_{i}\right| \\
\leq & \left|x_{0}\right| \omega_{i 0}\left(x_{0}, x_{1}\right) \vartheta+\sum_{j=1}^{i}\left|z_{j}\right| \\
& \cdot\left|x_{0}\right|^{i-j} \omega_{i j}\left(u_{0}, x_{0}, \bar{x}_{i}\right) \vartheta \\
= & +(n-i)\left|z_{i}\right| \omega_{0}\left(x_{0}\right) \vartheta \\
& \left.+\sum_{j=1}^{i}\left|\omega_{j}\right| \cdot\left|x_{0}\right|^{i-j} \omega_{i j}\left(x_{0}, z_{1}, x_{0}^{n-i}\right) \vartheta \quad z_{1} x_{0}^{n-i}, \ldots, z_{i} x_{0}^{n-i}\right) \vartheta \\
& +(n-i)\left|z_{i}\right| \omega_{0}\left(x_{0}\right) \vartheta .
\end{aligned}
$$

Introduce the notation

$$
\begin{aligned}
\widetilde{\omega}_{i 0}= & \omega_{i 0}\left(x_{0}, z_{1} x_{0}^{n-i}\right), \\
\widetilde{\omega}_{i j}= & \left|x_{0}\right|^{i-j} \omega_{i j} \\
& \times\left(u_{0}, x_{0}, z_{1} x_{0}^{n-i}, \ldots, z_{i} x_{0}^{n-i}\right), \quad 1 \leq i \leq j-1, \\
\widetilde{\omega}_{i i}= & \omega_{i i}\left(u_{0}, x_{0}, z_{1} x_{0}^{n-i}, \ldots, z_{i} x_{0}^{n-i}\right)+(n-i) \omega_{0}\left(x_{0}\right) .
\end{aligned}
$$

It is clear that $\widetilde{\omega}_{i j}(0 \leq j \leq i)$ are smooth nonnegative functions, and inequality (11) holds.

Lemma 7. For every $1 \leq i \leq n-1$, the following inequality holds:

$$
\begin{aligned}
\left|\widetilde{\varphi}_{i}\right| & \leq\left|z_{1}(t) z_{1}\left(t-\tau_{i}\right)\right| \Gamma_{i}\left(y(t), y\left(t-\tau_{i}\right)\right) \\
& \leq\left|z_{1}(t) z_{1}\left(t-\tau_{i}\right)\right| f_{i 1}(y(t)) f_{i 2}\left(y\left(t-\tau_{i}\right)\right),
\end{aligned}
$$

where $\Gamma_{i}, f_{i 1}$, and $f_{i 2}$ are smooth nonnegative functions.

Proof. According to Assumption 2, the nonlinear functions $\widetilde{\varphi}_{i}$ yield that

$$
\begin{aligned}
\left|\widetilde{\varphi}_{i}\right|= & \frac{\left|\varphi_{i}\left(y(t), y\left(t-\tau_{i}\right)\right)\right|}{\left|x_{0}^{n-i}(t)\right|} \\
\leq & \frac{\left|x_{1}(t) x_{1}\left(t-\tau_{i}\right)\right|}{\left|x_{0}^{n-i}(t)\right|} \bar{\varphi}_{i}\left(y(t), y\left(t-\tau_{i}\right)\right) \\
= & \left|z_{1}(t) x_{0}^{i-1}(t)\right| \\
& \cdot\left|z_{1}\left(t-\tau_{i}\right) x_{0}^{n-1}\left(t-\tau_{i}\right)\right| \bar{\varphi}_{i}\left(y(t), y\left(t-\tau_{i}\right)\right) .
\end{aligned}
$$

Let $\Gamma_{i}\left(y(t), y\left(t-\tau_{i}\right)\right)=|[1,0] y(t)|^{i-1}\left|[1,0] y\left(t-\tau_{i}\right)\right|^{n-1}$ $\bar{\varphi}_{i}\left(y(t), y\left(t-\tau_{i}\right)\right)$; then the above inequality can be expressed as

$$
\left|\widetilde{\varphi}_{i}\right| \leq\left|z_{1}(t) z_{1}\left(t-\tau_{i}\right)\right| \Gamma_{i}\left(y(t), y\left(t-\tau_{i}\right)\right) .
$$


It is seen that $\Gamma_{i}\left(y(t), y\left(t-\tau_{i}\right)\right)$ are smooth functions. Then using Lemma 5 , there exist smooth functions $f_{i 1} \geq 1$ and $f_{i 2} \geq 1$ such that

$$
\left|\widetilde{\varphi}_{i}\right| \leq\left|z_{1}(t) z_{1}\left(t-\tau_{i}\right)\right| f_{i 1}(y(t)) f_{i 2}\left(y\left(t-\tau_{i}\right)\right) .
$$

3.2. Control Design. In this section, we design the control inputs $u_{0}(t)$ and $u_{1}(t)$ subject to $x_{0}\left(t_{0}\right) \neq 0$. The case that the initial condition $x_{0}\left(t_{0}\right)=0$ will be treated in Section 4 . The design of the control inputs here is based on the backstepping method for the transformed system (10). The recursive procedure stops once the true system inputs occur.

Step 1. For the $x_{0}$-subsystem

$$
\dot{x}_{0}(t)=d_{0} u_{0}(t)+\phi_{0}\left(t, x_{0}(t), \theta\right),
$$

define new variables $r_{0}=1 /\left|d_{0}\right|, \widetilde{r}_{0}=r_{0}-\widehat{r}_{0}$, and $\widetilde{\vartheta}_{0}=\vartheta-\widehat{\vartheta}_{0}$, where $\widehat{r}_{0}$ and $\widehat{\vartheta}_{0}$ are the estimates of $r_{0}$ and $\vartheta$, respectively.

Consider the Lyapunov function candidate

$$
V_{0}=\frac{1}{2} x_{0}^{2}(t)+\frac{1}{2} \widetilde{\vartheta}_{0}^{2}+\frac{1}{2 r_{0}} \widetilde{r}_{0}^{2} .
$$

Calculating the time derivative of $V_{0}$ along the system (18)

$$
\dot{V}_{0}=x_{0}(t)\left[d_{0} u_{0}(t)+\phi_{0}\left(t, x_{0}(t), \theta\right)\right]-\widetilde{\vartheta}_{0} \dot{\hat{\vartheta}}_{0}-\frac{1}{r_{0}} \widetilde{r}_{0} \dot{\vec{r}}_{0} .
$$

The controller $u_{0}(t)$ can be chosen as

$$
u_{0}(t)=g_{0}\left(x_{0}, \widehat{\vartheta}_{0}, \widehat{r}_{0}\right) x_{0}(t),
$$

where

$$
\begin{aligned}
g_{0}\left(x_{0}, \widehat{\vartheta}_{0}, \widehat{r}_{0}\right) & =\operatorname{sign}\left(d_{0}\right) \widehat{r}_{0} \pi_{0}\left(x_{0}, \widehat{\vartheta}_{0}, \widehat{r}_{0}\right), \\
\pi_{0}\left(x_{0}, \widehat{\vartheta}_{0}, \widehat{r}_{0}\right)= & -\frac{1+\sqrt{1+\left(k_{0} / \widehat{r}_{0}\right)^{2}}}{2} \\
& \times\left[\omega_{0}\left(x_{0}\right) \widehat{\vartheta}_{0}+\sqrt{c_{0}^{2}+\left(\omega_{0}\left(x_{0}\right) \widehat{\vartheta}_{0}\right)^{2}}\right] .
\end{aligned}
$$

With the controller $u_{0}(t)$ in $(21)$, the time derivative of $V_{0}$ satisfies

$$
\begin{aligned}
\dot{V}_{0}= & \left(1-\frac{\widetilde{r}_{0}}{r_{0}}\right) \pi_{0}\left(x_{0}, \widehat{\vartheta}_{0}, \widehat{r}_{0}\right) x_{0}^{2}(t) \\
& +x_{0}(t) \phi_{0}\left(t, x_{0}(t), \theta\right)-\widetilde{\vartheta}_{0} \dot{\hat{\vartheta}}_{0}-\frac{1}{r_{0}} \widetilde{r}_{0} \dot{\hat{r}}_{0} \\
\leq & -\sqrt{c_{0}^{2}+\left(\omega_{0}\left(x_{0}\right) \widehat{\vartheta}_{0}\right)^{2}} x_{0}^{2}(t)-\frac{\widetilde{r}_{0}}{r_{0}} \pi_{0}\left(x_{0}, \widehat{\vartheta}_{0}, \widehat{r}_{0}\right) x_{0}^{2}(t) \\
& +x_{0}^{2}(t) \omega_{0}\left(x_{0}\right) \widetilde{\vartheta}_{0} \\
& -\widetilde{\vartheta}_{0} \dot{\hat{\vartheta}}_{0}-\frac{1}{r_{0}} \widetilde{r}_{0} \dot{\hat{r}}_{0} .
\end{aligned}
$$

Choosing the following update laws $\dot{\widehat{\vartheta}}_{0}$ and $\dot{\hat{r}}_{0}$ as

$$
\begin{gathered}
\dot{\widehat{\vartheta}}_{0}=\omega_{0}\left(x_{0}\right) x_{0}^{2}(t), \\
\dot{\widehat{r}}_{0}=-\pi_{0}\left(x_{0}, \widehat{\vartheta}_{0}, \widehat{r}_{0}\right) x_{0}^{2}(t),
\end{gathered}
$$

we have

$$
\dot{V}_{0} \leq-c_{0} x_{0}^{2}(t)
$$

where $c_{0}>0$ is a positive design parameter. Therefore, it implies that $x_{0}(t), \widehat{\vartheta}_{0}$, and $\widehat{r}_{0}$ are bound. By LaSalle's Invariant Theorem, we can further achieve that $x_{0}(t) \rightarrow 0$, as $t \rightarrow \infty$.

Remark 8. The closed-loop dynamics of $x_{0}$-subsystem is

$$
\dot{x}_{0}(t)=x_{0}(t)\left[d_{0} g_{0}\left(x_{0}, \widehat{\vartheta}_{0}, \widehat{r}_{0}\right)+\bar{\phi}_{0}\left(x_{0}, \theta\right)\right] \text {. }
$$

It is seen that $\psi(t)=d_{0} g_{0}\left(x_{0}, \widehat{\vartheta}_{0}, \widehat{r}_{0}\right)+\bar{\phi}_{0}\left(x_{0}, \theta\right)$ is bounded as $x_{0}(t), \widehat{\vartheta}_{0}, \widehat{r}_{0}$, and $\theta$ are bounded. On the other hand, the solution of $x_{0}$-system can be computed as

$$
x_{0}(t)=x_{0}\left(t_{0}\right) e^{\int_{t_{0}}^{t} \psi(s) d s} .
$$

Obviously, for $x_{0}\left(t_{0}\right) \neq 0$ and $t \geq t_{0}$, the solution $x_{0}(t)$ exists and satisfies $\left|x_{0}(t)\right|>0$. That is, $x_{0}(t)$ does not become zero at any time instant for $x_{0}\left(t_{0}\right) \neq 0$. Therefore, the introduced state scaling above is effective.

Under the controller $u_{0}(t)$ in (25), the $z$-system can be rewritten as

$$
\begin{aligned}
\dot{z}_{i}(t)= & d_{i} g_{0}\left(x_{0}, \widehat{\vartheta}_{0}, \widehat{r}_{0}\right) z_{i+1}(t) \\
& -(n-i) d_{0} g_{0}\left(x_{0}, \widehat{\vartheta}_{0}, \widehat{r}_{0}\right) z_{i}(t)+\widetilde{\phi}_{i}+\widetilde{\varphi}_{i}, \\
\dot{z}_{n}(t)= & d_{n} u_{1}(t)+\widetilde{\phi}_{n}+\widetilde{\varphi}_{n} .
\end{aligned}
$$

Step 2. For $z_{1}(t)$-subsystem in (30)

$$
\begin{aligned}
\dot{z}_{1}(t)= & d_{1} g_{0}\left(x_{0}, \widehat{\vartheta}_{0}, \widehat{r}_{0}\right) z_{2}(t) \\
& -(n-1) d_{0} g_{0}\left(x_{0}, \widehat{\vartheta}_{0}, \widehat{r}_{0}\right) z_{1}(t)+\widetilde{\phi}_{1}+\widetilde{\varphi}_{1},
\end{aligned}
$$

let $r_{1}=1 /\left|d_{1}\right|$, and $\widetilde{d}_{0}=d_{0}-\widehat{d}_{0}, \widetilde{d}_{1}=d_{1}-\widehat{d}_{1}, \widetilde{r}_{1}=r_{1}-\widehat{r}_{1}$, $\widetilde{\vartheta}_{1}=\vartheta-\widehat{\vartheta}_{1}$, and $\widetilde{v}=\vartheta^{2}-\widehat{v}$, where $\widehat{d}_{0}, \widehat{d}_{1}, \widehat{r}_{1}, \widehat{\vartheta}_{1}$, and $\widehat{v}$ are the estimates of unknown parameters $d_{0}, d_{1}, r_{1}, \vartheta$, and $\vartheta^{2}$, respectively. Introduce the coordinate transformations $\xi_{1}(t)=z_{1}(t)$ and $\xi_{2}(t)=z_{2}(t)-\alpha_{1}$, where $\alpha_{1}$ is regarded as the virtual control input. Construct the following LyapunovKrasovskii functional:

$$
\begin{aligned}
V_{1}= & V_{0}+\frac{1}{2} \xi_{1}^{2}(t)+\frac{1}{2} \widetilde{d}_{0}^{2}+\frac{1}{2} \widetilde{d}_{1}^{2}+\frac{1}{2 r_{1}} \widetilde{r}_{1}^{2}+\frac{1}{2} \widetilde{\vartheta}_{1}^{2}+\frac{1}{2} \widetilde{v}^{2} \\
& +\sum_{l=1}^{n} \sum_{k=1}^{l} \int_{t-\tau_{k}}^{t} \frac{\varepsilon_{l k}}{2} \xi_{1}^{2}(\sigma) f_{k 2}^{2}(y(\sigma)) d \sigma,
\end{aligned}
$$


where $\varepsilon_{l k}>0(1 \leq k \leq l \leq n)$ are scalars. Along (31), the time derivative of $V_{1}$ gives

$$
\begin{aligned}
& \dot{V}_{1}=\dot{V}_{0}+\xi_{1}(t) {\left[\left(\hat{d}_{1}+\tilde{d}_{1}\right) g_{0}\left(x_{0}, \widehat{\vartheta}_{0}, \widehat{r}_{0}\right) \xi_{2}(t)\right.} \\
&+d_{1} g_{0}\left(x_{0}, \widehat{\vartheta}_{0}, \widehat{r}_{0}\right) \alpha_{1}-(n-1)\left(\widehat{d}_{0}+\widetilde{d}_{0}\right) g_{0} \\
&\left.\times\left(x_{0}, \widehat{\vartheta}_{0}, \widehat{r}_{0}\right) z_{1}(t)+\widetilde{\varphi}_{1}+\widetilde{\phi}_{1}\right]-\widetilde{d}_{0} \dot{\hat{d}}_{0}-\widetilde{d}_{1} \dot{\hat{d}}_{1} \\
&-\frac{1}{r_{1}} \widetilde{r}_{1} \dot{\hat{r}}_{1}-\widetilde{\vartheta}_{1} \dot{\hat{\vartheta}}_{1}-\widetilde{v} \dot{\hat{v}}+\sum_{l=1}^{n} \sum_{k=1}^{l} \frac{\varepsilon_{l k}}{2} \xi_{1}^{2}(t) f_{k 2}^{2}(y(t)) \\
&-\sum_{l=1}^{n} \sum_{k=1}^{l} \frac{\varepsilon_{l k}}{2} \xi_{1}^{2}\left(t-\tau_{k}\right) f_{k 2}^{2}\left(y\left(t-\tau_{k}\right)\right) .
\end{aligned}
$$

By Lemmas 6 and 7, the following inequalities hold:

$$
\begin{aligned}
\left|\xi_{1}(t) \tilde{\phi}_{1}\right| \leq & x_{0}^{2}(t)+\xi_{1}^{2}(t) \gamma_{10}\left(x_{0}, z_{1}\right) \vartheta^{2} \\
& +\xi_{1}^{2}(t) \gamma_{11}\left(u_{0}, x_{0}, z_{1}\right) \vartheta, \\
\left|\xi_{1}(t) \widetilde{\varphi}_{1}\right| \leq \mid & \xi_{1}(t) z_{1}(t) z_{1}\left(t-\tau_{1}\right) \\
& \times f_{11}(y(t)) f_{12}\left(y\left(t-\tau_{1}\right)\right) \mid \\
\leq & \frac{1}{2 \varepsilon_{11}} \xi_{1}^{2}(t) z_{1}^{2}(t) f_{11}^{2}(y(t)) \\
& +\frac{\varepsilon_{11}}{2} \xi_{1}^{2}\left(t-\tau_{1}\right) f_{12}^{2}\left(y\left(t-\tau_{1}\right)\right),
\end{aligned}
$$

where $\gamma_{10}=(1 / 4) \widetilde{\omega}_{10}^{2}\left(x_{0}, z_{1}\right)$ and $\gamma_{11}=\widetilde{\omega}_{11}\left(u_{0}, x_{0}, z_{1}\right)$. Choose a virtual control function $\alpha_{1}\left(x_{0}, z_{1}, \widehat{\vartheta}_{0}, \widehat{\vartheta}_{1}, \widehat{v}, \widehat{r}_{0}, \widehat{r}_{1}, \widehat{d}_{0}\right)$ as follows:

$$
\begin{aligned}
& \alpha_{1}=\operatorname{sign}\left(d_{1}\right) \hat{r}_{1} \pi_{1}, \\
& \pi_{1}=-\frac{1}{g_{0}\left(x_{0}, \widehat{\vartheta}_{0}, \widehat{r}_{0}\right)}\left[c_{1} \xi_{1}(t)+\xi_{1}(t) \gamma_{10}\left(x_{0}, z_{1}\right) \widehat{v}\right. \\
&+\xi_{1}(t) \gamma_{11}\left(u_{0}, x_{0}, z_{1}\right) \widehat{\vartheta}_{1} \\
&+\frac{1}{2 \varepsilon_{11}} \xi_{1}(t) z_{1}^{2}(t) f_{11}^{2}(y(t)) \\
& \\
&\left.+\sum_{l=1}^{n} \sum_{k=1}^{l} \frac{\varepsilon_{l k}}{2} \xi_{1}(t) f_{k 2}^{2}(y(t))\right] \\
&+(n-1) \widehat{d}_{0} z_{1}(t),
\end{aligned}
$$

where $c_{1}$ is a positive design parameter. With the choice of the update law

$$
\dot{\widehat{r}}_{1}=-g_{0}\left(x_{0}, \widehat{\vartheta}_{0}, \widehat{r}_{0}\right) \pi_{1} \xi_{1}(t)
$$

we can obtain

$$
\begin{aligned}
\dot{V}_{1} \leq & -\left(c_{0}-1\right) x_{0}^{2}(t)-c_{1} \xi_{1}^{2}(t)+\widehat{d}_{1} g_{0}\left(x_{0}, \widehat{\vartheta}_{0}, \widehat{r}_{0}\right) \xi_{1}(t) \xi_{2}(t) \\
& -\widetilde{d}_{0}\left(\dot{\hat{d}}_{0}-\tau_{10}\right)-\widetilde{d}_{1}\left(\dot{\hat{d}}_{1}-\tau_{11}\right) \\
& -\widetilde{v}\left(\dot{\hat{v}}-\eta_{10}\right)-\widetilde{\vartheta}_{1}\left(\dot{\widehat{\vartheta}}_{1}-\eta_{11}\right) \\
& -\sum_{l=2}^{n} \sum_{k=1}^{l} \frac{\varepsilon_{l k}}{2} \xi_{1}^{2}\left(t-\tau_{k}\right) f_{k 2}^{2}\left(y\left(t-\tau_{k}\right)\right),
\end{aligned}
$$

where

$$
\begin{gathered}
\tau_{10}=\xi_{1}(t) h_{10}, \quad h_{10}=-(n-1) g_{0}\left(x_{0}, \widehat{\vartheta}_{0}, \widehat{r}_{0}\right) \xi_{1}(t), \\
\tau_{11}=\xi_{1}(t) h_{11}, \quad h_{11}=g_{0}\left(x_{0}, \widehat{\vartheta}_{0}, \widehat{r}_{0}\right) \xi_{2}(t), \\
\eta_{10}=\gamma_{10}\left(x_{0}, z_{1}\right) \xi_{1}^{2}(t), \quad \eta_{11}=\gamma_{11}\left(u_{0}, x_{0}, z_{1}\right) \xi_{1}^{2}(t) .
\end{gathered}
$$

Step $i(2 \leq i \leq n-1)$. Assume that, at step $i-1$, a virtual control function $\alpha_{i-1}$ and a Lyapunov functional $V_{i-1}$ have been designed for the $\left(z_{1}, \ldots, z_{i-1}\right)$-subsystem of (30) in such a way that

$$
\begin{aligned}
\dot{V}_{i-1} \leq & -\left(c_{0}-i+1\right) x_{0}^{2}(t)-\sum_{j=1}^{i-1}\left[c_{j}-i+j+1\right] \xi_{j}^{2}(t) \\
& +\widehat{d}_{i-1} g_{0}\left(x_{0}, \widehat{\vartheta}_{0}, \widehat{r}_{0}\right) \xi_{i-1}(t) \xi_{i}(t) \\
& -\sum_{j=0}^{i-1}\left(\tilde{d}_{j}+\sum_{l=j+2}^{i} \frac{\partial \alpha_{l-1}}{\partial \widehat{d}_{j}} \xi_{l}(t)\right)\left(\dot{\hat{d}}_{j}-\tau_{i j}\right) \\
& -\left(\widetilde{v}+\sum_{j=2}^{i-1} \frac{\partial \alpha_{j-1}}{\partial \widehat{v}} \xi_{j}(t)\right)\left(\dot{\hat{v}}-\eta_{i 0}\right) \\
& -\left(\widetilde{\vartheta}_{1}+\sum_{j=2}^{i-1} \frac{\partial \alpha_{j-1}}{\partial \widehat{\vartheta}_{1}} \xi_{j}(t)\right)\left(\dot{\hat{\vartheta}}_{1}-\eta_{i 1}\right) \\
& -\sum_{l=i}^{n} \sum_{k=1}^{l} \frac{1}{2} \xi_{1}^{2}\left(t-\tau_{k}\right) f_{k 2}^{2}\left(y\left(t-\tau_{k}\right)\right) .
\end{aligned}
$$

Now, we examine the $\left(z_{1}, \ldots, z_{i}\right)$-subsystem of (30). Define $r_{i}=1 /\left|d_{i}\right|$, and $\widetilde{d}_{i}=d_{i}-\widehat{d}_{i}$ and $\widetilde{r}_{i}=r_{i}-\widehat{r}_{i}$, where $\widehat{d}_{i}$ and $\widehat{r}_{i}$ are the estimates of unknown parameters $d_{i}$ and $r_{i}$, respectively. Introduce the coordinate transformations $\xi_{i+1}(t)=$ $z_{i+1}(t)-\alpha_{i}$, where $\alpha_{i}$ is regarded as a virtual control input, and construct the following Lyapunov-Krasovskii functional

$$
V_{i}=V_{i-1}+\frac{1}{2} \xi_{i}^{2}(t)+\frac{1}{2} \widetilde{d}_{i}^{2}+\frac{1}{2 r_{i}} \widetilde{r}_{i}^{2} .
$$


Based on (39), the time derivative of $V_{i}$ along the solutions of (30) satisfies

$$
\begin{aligned}
& \dot{V}_{i}=\dot{V}_{i-1}+\xi_{i}(t) \\
& \times\left\{\left(\widehat{d}_{i}+\widetilde{d}_{i}\right) g_{0}\left(x_{0}, \widehat{\vartheta}_{0}, \widehat{r}_{0}\right) \xi_{i+1}(t)\right. \\
& +d_{i} g_{0}\left(x_{0}, \widehat{\vartheta}_{0}, \widehat{r}_{0}\right) \alpha_{i}(t)+\widetilde{\varphi}_{i}+\widetilde{\phi}_{i} \\
& -(n-i)\left(\widehat{d}_{0}+\widetilde{d}_{0}\right) g_{0}\left(x_{0}, \widehat{\vartheta}_{0}, \widehat{r}_{0}\right) z_{i}(t) \\
& -\frac{\partial \alpha_{i-1}}{\partial x_{0}}\left[-\left(\widehat{d}_{0}+\widetilde{d}_{0}\right) g_{0}\left(x_{0}, \widehat{\vartheta}_{0}, \widehat{r}_{0}\right) x_{0}(t)+\phi_{0}\right] \\
& -\sum_{j=1}^{i-1} \frac{\partial \alpha_{i-1}}{\partial z_{j}}\left[-\left(\widehat{d}_{j}+\tilde{d}_{j}\right) g_{0}\left(x_{0}, \widehat{\vartheta}_{0}, \widehat{r}_{0}\right) z_{j+1}(t)\right. \\
& -(n-j)\left(\widehat{d}_{0}+\widetilde{d}_{0}\right) g_{0}\left(x_{0}, \widehat{\vartheta}_{0}, \widehat{r}_{0}\right) z_{j}(t) \\
& \left.+\widetilde{\varphi}_{j}+\tilde{\phi}_{j}\right] \\
& -\sum_{j=0}^{i-1} \frac{\partial \alpha_{i-1}}{\partial \widehat{r}_{j}} \dot{\hat{r}}_{j}-\sum_{j=0}^{i-2} \frac{\partial \alpha_{i-1}}{\partial \widehat{d}_{j}} \dot{\widehat{d}}_{j} \\
& \left.-\frac{\partial \alpha_{i-1}}{\partial \hat{\vartheta}_{0}} \dot{\widehat{\vartheta}}_{0}-\frac{\partial \alpha_{i-1}}{\partial \widehat{\vartheta}_{1}} \dot{\widehat{\vartheta}}_{1}-\frac{\partial \alpha_{i-1}}{\partial \widehat{v}} \dot{\hat{v}}\right\} \\
& -\widetilde{d}_{i} \dot{\hat{d}}_{i}-\frac{1}{r_{i}} \widetilde{r}_{i} \dot{\hat{r}}_{i} .
\end{aligned}
$$

By Lemmas 5 and 7 and Young inequality, the following inequality holds:

$$
\begin{aligned}
\mid \xi_{i}(t) & {\left[\widetilde{\varphi}_{i}-\sum_{j=1}^{i-1} \frac{\partial \alpha_{i-1}}{\partial z_{j}} \widetilde{\varphi}_{j}\right] \mid } \\
\leq & \frac{1}{2 \varepsilon_{i i}} \xi_{i}^{2}(t) z_{1}^{2}(t) f_{i 1}^{2}(y(t)) \\
& +\sum_{j=1}^{i-1} \frac{1}{2 \varepsilon_{i j}} \xi_{i}^{2}(t)\left(\frac{\partial \alpha_{i-1}}{\partial z_{j}}\right)^{2} z_{1}^{2}(t) f_{j 1}^{2}(y(t)) \\
& +\sum_{j=1}^{i} \frac{\varepsilon_{i j}}{2} \xi_{1}^{2}\left(t-\tau_{j}\right) f_{j 2}^{2}\left(y\left(t-\tau_{j}\right)\right) .
\end{aligned}
$$

Using Lemma 6 and Young inequality, there are nonnegative smooth functions $\gamma_{i 0}\left(x_{0}, \bar{z}_{i}, \widehat{\vartheta}_{0}, \widehat{\vartheta}_{1}, \widehat{v}, \widehat{r}_{0}, \ldots, \widehat{r}_{i-1}\right.$, $\left.\widehat{d}_{0}, \ldots, \widehat{d}_{i-2}\right)$ and $\gamma_{i 1}\left(u_{0}, x_{0}, \bar{z}_{i}\right)$ such that

$$
\begin{aligned}
& \left|\xi_{i}(t)\left[\tilde{\phi}_{i}-\sum_{j=1}^{i-1} \frac{\partial \alpha_{i-1}}{\partial z_{j}} \tilde{\phi}_{j}-\frac{\partial \alpha_{i-1}}{\partial x_{0}} \phi_{0}\right]\right| \\
& \quad \leq x_{0}^{2}(t)+\sum_{j=1}^{i-1} \xi_{j}^{2}(t)+\xi_{i}^{2}(t) \gamma_{i 0} \vartheta^{2}+\xi_{i}^{2}(t) \gamma_{i 1} \vartheta
\end{aligned}
$$

where $\gamma_{i 0}$ and $\gamma_{i 1}$ are known nonnegative functions.
Choose the following virtual control function $\alpha_{i}$ :

$$
\begin{aligned}
& \alpha_{i}=\operatorname{sign}\left(d_{i}\right) \widehat{r}_{i} \pi_{i}, \\
& \pi_{i}=-\widehat{d}_{i-1} \xi_{i-1}(t)+(n-i) \widehat{d}_{0} z_{i}(t) \\
& +\sum_{j=1}^{i-1} \widehat{d}_{j} \frac{\partial \alpha_{i-1}}{\partial z_{j}} z_{j+1}(t)-\widehat{d}_{0} \sum_{j=1}^{i-1}(n-j) \frac{\partial \alpha_{i-1}}{\partial z_{j}} z_{j}(t) \\
& +\widehat{d}_{0} \frac{\partial \alpha_{i-1}}{\partial x_{0}} x_{0}(t)-\frac{1}{g_{0}\left(x_{0}, \widehat{\vartheta}_{0}, \widehat{r}_{0}\right)} \\
& \times\left[c_{i} \xi_{i}(t)+\xi_{i}(t) \gamma_{i 0} \widehat{v}+\xi_{i}(t) \gamma_{i 1} \widehat{\vartheta}_{1}\right. \\
& -\frac{\partial \alpha_{i-1}}{\partial \widehat{\vartheta}_{0}}-\sum_{j=0}^{i-1} \frac{\partial \alpha_{i-1}}{\partial \widehat{\hat{\vartheta}}_{j}} \dot{\hat{r}}_{j} \\
& -\sum_{j=0}^{i-2} \frac{\partial \alpha_{i-1}}{\partial \widehat{d}_{j}} \tau_{i j}-\frac{\partial \alpha_{i-1}}{\partial \widehat{v}} \eta_{i 0}-\frac{\partial \alpha_{i-1}}{\partial \widehat{\vartheta}_{1}} \eta_{i 1} \\
& +\frac{1}{2 \varepsilon_{i i}} \xi_{i}(t) z_{1}^{2}(t) f_{i 1}^{2}(y(t)) \\
& +\sum_{j=1}^{i-1} \frac{1}{2 \varepsilon_{i j}} \xi_{i}(t)\left(\frac{\partial \alpha_{i-1}}{\partial z_{j}}\right)^{2} z_{1}^{2}(t) f_{j 1}^{2}(y(t)) \\
& -\sum_{l=2}^{i-1} \sum_{j=0}^{l-2} \xi_{l}(t) \frac{\partial \alpha_{l-1}}{\partial \widehat{d}_{j}} h_{i j}-\sum_{j=2}^{i-1} \xi_{j}(t) \frac{\partial \alpha_{j-1}}{\partial \widehat{v}} \gamma_{i 0} \\
& \left.-\sum_{j=2}^{i-1} \xi_{j}(t) \frac{\partial \alpha_{j-1}}{\partial \widehat{\vartheta}_{1}} \gamma_{i 1}\right]
\end{aligned}
$$

where $c_{i}$ is a positive design parameter and $\tau_{i j}(0 \leq j \leq i-2)$, $\eta_{i 0}$ and $\eta_{i 1}$ are pending nonnegative functions to be specified in (47). Moreover, construct the following update law:

$$
\dot{\widehat{r}}_{i}=-g_{0}\left(x_{0}, \widehat{\vartheta}_{0}, \widehat{r}_{0}\right) \pi_{i} \xi_{i}(t)
$$

Substituting inequalities (42)-(45) into (41) yields

$$
\begin{aligned}
\dot{V}_{i} \leq & -\left(c_{0}-i\right) x_{0}^{2}(t)-\sum_{j=1}^{i}\left[c_{j}-i+j\right] \xi_{j}^{2}(t) \\
& +\widehat{d}_{i} g_{0}\left(x_{0}, \widehat{\vartheta}_{0}, \widehat{r}_{0}\right) \xi_{i}(t) \xi_{i+1}(t) \\
& -\sum_{j=0}^{i}\left(\tilde{d}_{j}+\sum_{l=j+2}^{i} \frac{\partial \alpha_{l-1}}{\partial \widehat{d}_{j}} \xi_{l}(t)\right)\left(\dot{\hat{d}}_{j}-\tau_{i j}\right) \\
& -\left(\tilde{v}+\sum_{j=2}^{i} \frac{\partial \alpha_{j-1}}{\partial \widehat{v}} \xi_{j}(t)\right)\left(\dot{\hat{v}}-\eta_{i 0}\right)
\end{aligned}
$$




$$
\begin{aligned}
& -\left(\widetilde{\vartheta}_{1}+\sum_{j=2}^{i} \frac{\partial \alpha_{j-1}}{\partial \widehat{\vartheta}_{1}} \xi_{j}(t)\right)\left(\dot{\hat{\vartheta}}_{1}-\eta_{i 1}\right) \\
& -\sum_{l=i+1}^{n} \sum_{k=1}^{l} \frac{\varepsilon_{l k}}{2} \xi_{1}^{2}\left(t-\tau_{k}\right) f_{k 2}^{2}\left(y\left(t-\tau_{k}\right)\right),
\end{aligned}
$$

where

$$
\begin{aligned}
\tau_{i 0}= & \tau_{i-1,0}+h_{i 0} \xi_{i}(t), \\
h_{i 0}= & -(n-i) g_{0}\left(x_{0}, \widehat{\vartheta}_{0}, \widehat{r}_{0}\right) z_{i}(t) \\
& -\frac{\partial \alpha_{i-1}}{\partial x_{0}} g_{0}\left(x_{0}, \widehat{\vartheta}_{0}, \widehat{r}_{0}\right) x_{0}(t) \\
& +\sum_{j=1}^{i-1}(n-j) \frac{\partial \alpha_{i-1}}{\partial z_{j}} g_{0}\left(x_{0}, \widehat{\vartheta}_{0}, \widehat{r}_{0}\right) z_{j}(t), \\
& \tau_{i l}=\tau_{i-1, l}+h_{i l} \xi_{i}(t), \\
& h_{i l}=-\frac{\partial \alpha_{i-1}}{\partial z_{l}} g_{0}\left(x_{0}, \widehat{\vartheta}_{0}, \widehat{r}_{0}\right) z_{l+1}(t), \\
\tau_{i i}= & h_{i i} \xi_{i}(t), \quad 1 \leq l \leq i-1, \\
\eta_{i 0}= & \eta_{i-1,0}+\gamma_{i 0} \xi_{i}(t), \quad \eta_{i 1}=g_{0}\left(x_{0}, \widehat{\vartheta}_{0}, \widehat{r}_{0}\right) \xi_{i+1}(t), \gamma_{i 1} \xi_{i}(t) .
\end{aligned}
$$

Step $n$. At the last step, we study the whole $z$-subsystem (30), and the true input $u_{1}(t)$ will be designed on the basis of the virtual control $\alpha_{i^{\prime}} s$ and the Lyapunov function $V_{n-1}$ introduced before. Here, let us consider a LyapunovKrasovskii function $V_{n}$ as follows:

$$
V_{n}=V_{n-1}+\frac{1}{2} \xi_{n}^{2}(t)+\frac{1}{2 r_{n}} \widetilde{r}_{n}^{2}
$$

Denote $r_{n}=1 /\left|d_{n}\right|$ and $\widetilde{r}_{n}=r_{n}-\widehat{r}_{n}$, where $\widehat{r}_{n}$ is the estimate of unknown parameter $r_{n}$. Recall that $\xi_{n}(t)=z_{n}(t)-\alpha_{n-1}$, with $\alpha_{n-1}$ being a virtual control input, then

$$
\begin{aligned}
\dot{V}_{n-1} \leq & -\left(c_{0}-n+1\right) x_{0}^{2}(t)-\sum_{j=1}^{n-1}\left[c_{j}-n+j+1\right] \xi_{j}^{2}(t) \\
& +\widehat{d}_{n-1} g_{0}\left(x_{0}, \widehat{\vartheta}_{0}, \widehat{r}_{0}\right) \xi_{n-1}(t) \xi_{n}(t) \\
& -\sum_{j=0}^{n-1}\left(\tilde{d}_{j}+\sum_{l=j+2}^{n-1} \frac{\partial \alpha_{l-1}}{\partial \widehat{d}_{j}} \xi_{l}(t)\right)\left(\dot{\hat{d}}_{j}-\tau_{n-1, j}\right) \\
& -\left(\widetilde{v}+\sum_{j=2}^{n-1} \frac{\partial \alpha_{j-1}}{\partial \widehat{v}} \xi_{j}(t)\right)\left(\dot{\hat{v}}-\eta_{n-1,0}\right)
\end{aligned}
$$

$$
\begin{aligned}
& -\left(\widetilde{\vartheta}_{1}+\sum_{j=2}^{n-1} \frac{\partial \alpha_{j-1}}{\partial \widehat{\vartheta}_{1}} \xi_{j}(t)\right)\left(\dot{\widehat{\vartheta}}_{1}-\eta_{n-1,1}\right) \\
& -\sum_{k=1}^{n} \frac{\varepsilon_{l k}}{2} \xi_{1}^{2}\left(t-\tau_{k}\right) f_{k 2}^{2}\left(y\left(t-\tau_{k}\right)\right) .
\end{aligned}
$$

Differentiating $V_{n}$ along the solution of (30) gives

$$
\begin{aligned}
& \dot{V}_{n}=\dot{V}_{n-1}+\xi_{n}(t) \\
& \times\left\{d_{n} u_{1}(t)+\widetilde{\varphi}_{n}+\tilde{\phi}_{n}-\frac{\partial \alpha_{n-1}}{\partial x_{0}}\right. \\
& \times\left[\left(\widehat{d}_{0}+\widetilde{d}_{0}\right) g_{0}\left(x_{0}, \widehat{\vartheta}_{0}, \widehat{r}_{0}\right) x_{0}(t)+\phi_{0}\right] \\
& -\sum_{j=1}^{n-1} \frac{\partial \alpha_{n-1}}{\partial z_{j}}\left[\left(\widehat{d}_{j}+\tilde{d}_{j}\right) g_{0}\left(x_{0}, \widehat{\vartheta}_{0}, \widehat{r}_{0}\right) z_{j+1}(t)\right. \\
& -(n-j)\left(\widehat{d}_{0}+\widetilde{d}_{0}\right) g_{0} \\
& \left.\times\left(x_{0}, \widehat{\vartheta}_{0}, \widehat{r}_{0}\right) z_{j}(t)+\widetilde{\varphi}_{j}+\widetilde{\phi}_{j}\right] \\
& -\sum_{j=0}^{n-1} \frac{\partial \alpha_{n-1}}{\partial \widehat{r}_{j}} \dot{\hat{r}}_{j}-\sum_{j=0}^{n-2} \frac{\partial \alpha_{i-1}}{\partial \widehat{d}_{j}} \dot{\hat{d}}_{j} \\
& \left.-\frac{\partial \alpha_{n-1}}{\partial \widehat{\vartheta}_{0}}-\frac{\partial \alpha_{n-1}}{\partial \widehat{\vartheta}_{1}}-\frac{\partial \alpha_{n-1}}{\partial \widehat{v}} \dot{\hat{v}}\right\} \\
& -\frac{1}{r_{i}} \widetilde{r}_{n} \dot{\hat{r}}_{n}
\end{aligned}
$$

Similarly, by Lemmas 5-7 and Young inequality, we can easily obtain that there are scalars $\varepsilon_{n j}>0(1 \leq j \leq n)$ and smooth nonnegative functions $\gamma_{n 0}$ and $\gamma_{n 1}$ such that

$$
\begin{aligned}
\mid \xi_{n}(t) & {\left[\widetilde{\varphi}_{n}-\sum_{j=1}^{n-1} \frac{\partial \alpha_{n-1}}{\partial z_{j}} \widetilde{\varphi}_{j}\right] \mid } \\
\leq & \frac{1}{2 \varepsilon_{n n}} \xi_{n}^{2}(t) z_{1}^{2}(t) f_{n 1}^{2}(y(t)) \\
& +\sum_{j=1}^{n-1} \frac{1}{2 \varepsilon_{n j}} \xi_{n}^{2}(t)\left(\frac{\partial \alpha_{n-1}}{\partial z_{j}}\right)^{2} z_{1}^{2}(t) f_{j 1}^{2}(y(t)) \\
& +\sum_{j=1}^{n} \frac{\varepsilon_{n j}}{2} \xi_{1}^{2}\left(t-\tau_{j}\right) f_{j 2}^{2}\left(y\left(t-\tau_{j}\right)\right),
\end{aligned}
$$




$$
\begin{gathered}
\left|\xi_{n}(t)\left[\tilde{\phi}_{n}-\sum_{j=1}^{n-1} \frac{\partial \alpha_{n-1}}{\partial z_{j}} \widetilde{\phi}_{j}-\frac{\partial \alpha_{n-1}}{\partial x_{0}} \phi_{0}\right]\right| \\
\leq x_{0}^{2}(t)+\sum_{j=1}^{n-1} \xi_{j}^{2}(t)+\xi_{n}^{2}(t) \gamma_{n 0} \vartheta^{2} \\
+\xi_{n}^{2}(t) \gamma_{n 1}\left(u_{0}, x_{0}, \bar{z}_{n}\right) \vartheta .
\end{gathered}
$$

Next, we can design the control input $u_{1}(t)$ as follows:

$$
\begin{aligned}
& u_{1}(t)=\operatorname{sign}\left(d_{n}\right) \widehat{r}_{n} \pi_{n}, \\
& \pi_{n}=-g_{0}\left(x_{0}, \widehat{\vartheta}_{0}, \widehat{r}_{0}\right) \\
& \times\left\{\widehat{d}_{n-1} \xi_{n-1}(t)-\sum_{j=1}^{n-1} \frac{\partial \alpha_{n-1}}{\partial z_{j}}\right. \\
& \times\left[\widehat{d}_{j} z_{j+1}(t)-(n-j) \widehat{d}_{0} z_{j}(t)\right] \\
& \left.-\widehat{d}_{0} \frac{\partial \alpha_{n-1}}{\partial x_{0}} x_{0}(t)\right\}-c_{n} \xi_{n}(t)-\xi_{n}(t) \gamma_{n 0} \widehat{v} \\
& -\xi_{n}(t) \gamma_{n 1} \widehat{\vartheta}_{1}+\frac{\partial \alpha_{n-1}}{\partial \widehat{\vartheta}_{0}} \dot{\widehat{\vartheta}}_{0}+\sum_{j=0}^{n-1} \frac{\partial \alpha_{n-1}}{\partial \widehat{r}_{j}} \dot{\widehat{r}}_{j} \\
& +\sum_{j=0}^{n-2} \frac{\partial \alpha_{n-1}}{\partial \widehat{d}_{j}} \tau_{n j}+\frac{\partial \alpha_{n-1}}{\partial \widehat{v}} \eta_{n 0}+\frac{\partial \alpha_{n-1}}{\partial \widehat{\vartheta}_{1}} \eta_{n 1} \\
& -\frac{1}{2 \varepsilon_{n n}} \xi_{n}(t) z_{1}^{2}(t) f_{n 1}^{2}(y(t)) \\
& -\sum_{j=1}^{n-1} \frac{1}{2 \varepsilon_{n j}} \xi_{n}(t)\left(\frac{\partial \alpha_{n-1}}{\partial z_{j}}\right)^{2} z_{1}^{2}(t) f_{j 1}^{2}(y(t)) \\
& +\sum_{l=2}^{n-1} \sum_{j=0}^{l-2} \xi_{l}(t) \frac{\partial \alpha_{l-1}}{\partial \widehat{d}_{j}} h_{n j}+\sum_{j=2}^{n-1} \xi_{j}(t) \frac{\partial \alpha_{j-1}}{\partial \widehat{v}} \eta_{n 0} \\
& +\sum_{j=2}^{n-1} \xi_{j}(t) \frac{\partial \alpha_{j-1}}{\partial \widehat{\vartheta}_{1}} \eta_{n 1}
\end{aligned}
$$

where $c_{n}$ is a positive design parameter and $\tau_{n j}(0 \leq j \leq$ $n-1), \eta_{n 0}$ and $\eta_{n 1}$ are smooth nonnegative functions to be specified in (56). With the choice of the update law

$$
\dot{\hat{r}}_{n}=-\pi_{n} \xi_{i}(t)
$$

it renders

$$
\begin{aligned}
\dot{V}_{n} \leq & -\left(c_{0}-n\right) x_{0}^{2}(t)-\sum_{j=1}^{n}\left[c_{j}-n+j\right] \xi_{j}^{2}(t) \\
& -\sum_{j=0}^{n-1}\left(\widetilde{d}_{j}+\sum_{l=j+2}^{n-1} \frac{\partial \alpha_{l-1}}{\partial \widehat{d}_{j}} \xi_{l}(t)\right)\left(\dot{\hat{d}}_{j}-\tau_{n j}\right) \\
& -\left(\tilde{v}+\sum_{j=2}^{n} \frac{\partial \alpha_{j-1}}{\partial \widehat{v}} \xi_{j}(t)\right)\left(\dot{\hat{v}}-\eta_{n 0}\right) \\
& -\left(\widetilde{\vartheta}_{1}+\sum_{j=2}^{n} \frac{\partial \alpha_{j-1}}{\partial \widehat{\vartheta}_{1}} \xi_{j}(t)\right)\left(\dot{\hat{\vartheta}}_{1}-\eta_{n 1}\right),
\end{aligned}
$$

where

$$
\begin{aligned}
\tau_{n 0}= & \tau_{n-1,0}+h_{n 0} \xi_{n}(t), \\
h_{n 0}= & -\frac{\partial \alpha_{n-1}}{\partial x_{0}} g_{0}\left(x_{0}, \widehat{\vartheta}_{0}, \widehat{r}_{0}\right) x_{0}(t) \\
& +\sum_{j=1}^{n-1}(n-j) \frac{\partial \alpha_{n-1}}{\partial z_{j}} g_{0}\left(x_{0}, \widehat{\vartheta}_{0}, \widehat{r}_{0}\right) z_{j}(t), \\
& \tau_{n l}=\tau_{n-1, l}+h_{n l} \xi_{i}(t), \quad 1 \leq l \leq n-1, \\
& h_{n l}=-\frac{\partial \alpha_{n-1}}{\partial z_{l}} g_{0}\left(x_{0}, \widehat{\vartheta}_{0}, \widehat{r}_{0}\right) z_{l+1}(t), \\
\eta_{n 0}= & \eta_{n-1,0}+\gamma_{n 0} \xi_{n}(t), \quad \eta_{n 1}=\eta_{n-1,1}+\gamma_{n 1} \xi_{n}(t) .
\end{aligned}
$$

Furthermore, employing the following update laws

$$
\dot{\hat{d}}_{j}=\tau_{n j}, \quad 0 \leq j \leq n-1, \quad \dot{\hat{v}}=\eta_{n 0}, \quad \dot{\widehat{\vartheta}}_{1}=\eta_{n 1}
$$

eventually achieves

$$
\dot{V}_{n} \leq-\left(c_{0}-n\right) x_{0}^{2}(t)-\sum_{j=1}^{n}\left[c_{j}-n+j\right] \xi_{j}^{2}(t) .
$$

This together with (48) implies that $x_{0}, \xi_{i}, \widetilde{v}, \widetilde{\vartheta}_{0}, \widetilde{\vartheta}_{1}$ and $\tilde{d}_{i}, \widetilde{r}_{i}(0 \leq i \leq n)$ are bounded. Since $\theta$ and $d_{i}$ are constant vector and constant, respectively, we know that $\widehat{\vartheta}_{0}, \widehat{\vartheta}_{1}$ and $\widehat{d}_{i}$, $\widehat{r}_{i}(0 \leq i \leq n)$ are also bounded. According to the definitions of the virtual control input $\alpha_{i}$ in the above design procedure, $z_{i}$ are bounded as $\alpha_{i}$ are bounded. It indicates that all signals of the closed loop system are bounded.

LaSalle Invariant Theorem further achieves that $x_{0}, \xi_{i}, \widetilde{v}, \widetilde{\vartheta}_{0}, \widetilde{\vartheta}_{1}, \widetilde{d}_{i}, \widetilde{r}_{i} \rightarrow 0$ as $t \rightarrow \infty$. The boundedness of all signals and the choice of virtual control functions $\alpha_{i}$ imply that $\alpha_{i}$ converge to zero, which shows that $z_{i}$ also tend to zero. From the transformation $x_{i}=z_{i} x_{0}^{n-i}$, we can prove that $x_{0} \rightarrow 0$, as $t \rightarrow 0$.

The above analysis is summarized into the following theorem. 
Theorem 9. For the system (1), under Assumptions 1 and 2, if the control strategies (21) and (52) are applied with an appropriate choice of the design parameters $c_{i}(0 \leq i \leq n)$, the global asymptotic stabilization of the closed loop system is achieved for $x_{0}\left(t_{0}\right) \neq 0$.

In the next section, we will deal with the stability analysis of the closed loop system with our control laws (21) and (52) as long as the initial condition $x_{0}\left(t_{0}\right)$ is zero.

\section{Switching Controller}

Several switching controllers have been proposed in some existing literature resources. As well known, the choice of a constant feedback for $u_{0}(t)$ may lead to a finite escape. That is, the solution $x_{0}(t)$ issued from the origin may blow up before the switch. Usually, the phenomenon occurs for systems with non-Lipschitz nonlinearities. In this paper, the term $\phi_{0}\left(t, x_{0}(t), \theta\right)$ in $x_{0}(t)$-subsystem does not satisfy the Lipschitz conditions; then we should apply a novel switching control design. When the initial state $x_{0}\left(t_{0}\right)=0$, choose controller $u_{0}(t)$ as

$$
u_{0}(t)=g_{0}\left(x_{0}, \widehat{\vartheta}_{0}, \widehat{r}_{0}\right) x_{0}(t)+\operatorname{sign}\left(d_{0}\right) \lambda, \quad t \in\left[t_{0}, t_{s}\right],
$$

where $\lambda>0$ is a constant, $g_{0}\left(x_{0}, \widehat{\vartheta}_{0}, \widehat{r}_{0}\right)$ is defined in (22), and update laws of the parameters $\widehat{\vartheta}_{0}$ and $\widehat{r}_{0}$ are chosen as in (25) and (26), respectively.

With controller $u_{0}(t)$ in (59), the derivative of the Lyapunov function $V_{0}$ in (19) along $x_{0}$-subsystem gives that

$$
\begin{aligned}
\dot{V}_{0} & \leq-c_{0} x_{0}^{2}(t)+\lambda\left|d_{0}\right| x_{0}(t) \\
& =-c_{0}\left[x_{0}(t)-\frac{\lambda\left|d_{0}\right|}{2 c_{0}}\right]^{2}+\frac{\lambda^{2}\left|d_{0}\right|^{2}}{4 c_{0}^{2}} .
\end{aligned}
$$

The above inequality indicates that $x_{0}(t), \widehat{\vartheta}_{0}$, and $\widehat{r}_{0}$ are bounded; then state $x_{0}(t)$ cannot blow up during the time period $\left[t_{0}, t_{s}\right)$.

For $x_{0}(t)=0$ and Assumption 2, we have

$$
\begin{aligned}
\dot{x}_{0}(t) & =d_{0} u_{0}(t)+\phi_{0}\left(t, x_{0}(t), \theta\right) \\
& \geq-\left[-d_{0} g_{0}\left(x_{0}, \widehat{\vartheta}_{0}, \widehat{r}_{0}\right)+\omega_{0}\left(x_{0}\right) \vartheta\right] x_{0}(t)+\lambda\left|d_{0}\right| \\
& =-\omega_{0}(t)+\lambda\left|d_{0}\right|
\end{aligned}
$$

where $\omega=-d_{0} g_{0}\left(x_{0}, \widehat{\vartheta}_{0}, \widehat{r}_{0}\right)+\omega_{0}\left(x_{0}\right) \vartheta$. Obviously, $\omega$ is bounded.

The above inequality indicates that

$$
\begin{aligned}
x_{0}(t) \geq & e^{-\int_{t_{0}}^{t} \omega(s) d s} \int_{t_{0}}^{t} \lambda\left|d_{0}\right| e^{-\int_{t_{0}}^{s} \omega(\tau) d \tau} d s \\
& +x_{0}\left(t_{0}\right) e^{-\int_{t_{0}}^{t} \omega(s) d s} \\
= & e^{-\int_{t_{0}}^{t} \omega(s) d s} \int_{t_{0}}^{t} \lambda\left|d_{0}\right| e^{-\int_{t_{0}}^{s} \omega(\tau) d \tau} d s .
\end{aligned}
$$

It is clear that when $0<t \leq t_{s},\left|x_{0}(t)\right|>0$. Therefore, the state scaling coordinate transformation in (8) is effective, and we can use the following switching control strategy for $x_{0}\left(t_{0}\right)=0$.

During the time period $\left[t_{0}, t_{s}\right]$, using the controller $u_{0}(t)$ in (59), when $t=t_{0}$ and $u_{0}(t)$ is a constant, the controller $u_{1}(t)$ can be designed implying the simple nonlinear backstepping method. When $t_{0}<t \leq t_{s}$, choose the controller $u_{1}(t)$ as the iterative procedure in Section 3. Since $x_{0}\left(t_{s}\right) \neq 0$ at $t_{s}$, we switch the control law $u_{0}(t)$ and $u_{1}(t)$ into (21) and (52), respectively.

Theorem 10. For the system (1), under Assumptions 1 and 2 , if the above switching control strategy is applied with an appropriate choice of the design parameters $c_{i}(0 \leq i \leq n)$, then the closed-loop system is globally asymptotically regulated at the origin for $x_{0}\left(t_{0}\right)=0$.

\section{Simulation Example}

In this section, a numerical example will be given to illustrate that the proposed systematic control law design method is effective.

Example 1. Consider the following system:

$$
\begin{aligned}
\dot{x}_{0}(t)= & d_{0} u_{0}(t)+\left(x_{0}(t)\right)^{\theta_{0}} \\
\dot{x}_{1}(t)= & d_{1} u_{0}(t) x_{2}(t)+\frac{1}{2} \ln \left(1+x_{1}^{2}(t)\right) e^{x_{0}(t)} x_{1}^{2}(t-0.3) \\
& +x_{1}(t) \theta_{1}^{x_{1}(t)} \\
\dot{x}_{2}(t)= & d_{2} u_{1}(t)+x_{1}(t) e^{x_{0}(t-0.2)} x_{1}^{3 / 2}(t-0.2) \\
& +\ln \left(1+\left(\theta_{2} x_{2}(t)\right)^{2}\right)
\end{aligned}
$$

where $d_{0}, d_{1}$, and $d_{2}$ are unknown virtual control coefficients, and $\theta_{0}, \theta_{1}$, and $\theta_{2}$ are unknown bounded parameters. Our purpose is to design controllers $u_{0}(t)$ and $u_{1}(t)$ such that the states of the closed-loop system tend to zero when $t \rightarrow \infty$.

To apply the proposed design method, we make the estimation of nonlinear functions in system (62) as follows:

$$
\begin{gathered}
\left(x_{0}(t)\right)^{\theta_{0}} \leq\left|x_{0}(t)\right| e^{\left(\theta_{0}-1\right) \ln \left|x_{0}(t)\right|} \\
\leq\left|x_{0}(t)\right| e^{(1 / 2)\left(\theta_{0}-1\right)^{2}+(1 / 2) \ln ^{2}\left|x_{0}(t)\right|} \\
\leq\left|x_{0}(t)\right| e^{(1 / 8) \ln ^{2}\left(1+x_{0}^{2}(t)\right)} \cdot e^{(1 / 2)\left(\theta_{0}-1\right)}, \\
x_{1}(t) \theta_{1}^{x_{1}(t)} \leq\left|x_{1}(t)\right| e^{x_{1}(t) \ln \theta_{1}} \leq\left|x_{1}(t)\right| e^{(1 / 2) x_{1}^{2}(t)} e^{(1 / 2) \ln ^{2} \theta_{1}}, \\
\ln \left(1+\left(\theta_{2} x_{2}(t)\right)^{2}\right) \leq\left|x_{2}(t)\right| \cdot\left|\theta_{2}\right| .
\end{gathered}
$$


Let $\vartheta=e^{(1 / 2)\left(\theta_{0}-1\right)}+e^{(1 / 2)\left(\theta_{0}-1\right)}+\left|\theta_{2}\right|$; then the above inequalities can be deduced as

$$
\begin{gathered}
\left(x_{0}(t)\right)^{\theta_{0}} \leq\left|x_{0}(t)\right| e^{(1 / 8) \ln ^{2}\left(1+x_{0}^{2}(t)\right)} \vartheta \\
x_{1}(t) \theta_{1}^{x_{1}(t)} \leq\left|x_{1}(t)\right| e^{(1 / 2) x_{1}^{2}(t)} \vartheta \\
\ln \left(1+\left(\theta_{2} x_{2}(t)\right)^{2}\right) \leq\left|x_{2}(t)\right| \vartheta
\end{gathered}
$$

Introduce the following coordinate transformation:

$$
z_{1}(t)=\frac{x_{1}(t)}{x_{0}(t)}, \quad z_{2}(t)=x_{2}(t)
$$

then system (62) can be rewritten as

$$
\begin{gathered}
\dot{x}_{0}(t)=d_{0} u_{0}(t)+\left(x_{0}(t)\right)^{\theta_{0}}, \\
\dot{z}_{1}(t)=\frac{u_{0}(t)}{x_{0}(t)} d_{1} z_{2}(t)-\frac{u_{0}(t)}{x_{0}(t)} d_{0} z_{1}(t) \\
+\frac{x_{1}(t) \theta_{1}^{x_{1}(t)}}{x_{0}(t)}-\frac{\left(x_{0}(t)\right)^{\theta_{0}}}{x_{0}(t)} z_{1}(t) \\
\times \frac{(1 / 2) \ln \left(1+x_{1}^{2}(t)\right) e^{x_{0}(t)} x_{1}^{2}(t-0.3)}{x_{0}(t)}, \\
\dot{z}_{2}(t)=d_{2} u_{1}(t)+x_{1}(t) e^{x_{0}(t-0.2)} x_{1}^{3}(t-0.2) \\
+\ln \left(1+\left(\theta_{2} x_{2}(t)\right)^{2}\right) .
\end{gathered}
$$
$u_{0}(t)$

For $x_{0}(t)$-subsystem, design the following controller

$$
u_{0}(t)=g_{0}\left(x_{0}, \widehat{\vartheta}_{0}, \widehat{r}_{0}\right) x_{0}(t)
$$

with

$$
\begin{gathered}
g_{0}=\operatorname{sign}\left(d_{0}\right) \widehat{r}_{0} \pi_{0}, \\
\pi_{0}=-\frac{1+\sqrt{1+\left(k_{0} / \widehat{r}_{0}\right)^{2}}}{2}\left[\omega_{0} \widehat{\vartheta}_{0}+\sqrt{c_{0}^{2}+\left(\omega_{0} \widehat{\vartheta}_{0}\right)^{2}}\right],
\end{gathered}
$$

where $\omega_{0}=e^{(1 / 8) \ln ^{2}\left(1+x_{0}^{2}(t)\right)}$ and $k_{0}$, and $c_{0}$ are design constants. Moreover, the adaptation laws of $\widehat{r}_{0}$ and $\widehat{\vartheta}_{0}$ are chosen as

$$
\begin{gathered}
\dot{\widehat{\vartheta}}_{0}=\omega_{0} x_{0}^{2}(t), \\
\dot{\widehat{r}}_{0}=-\pi_{0} x_{0}^{2}(t) .
\end{gathered}
$$

Define $\xi_{1}(t)=z_{1}(t)$ and $\xi_{2}(t)=z_{2}(t)-\alpha_{1}$; according to the design procedure in Section 3, the following controller $u_{1}(t)$ can be given:

$$
u_{1}(t)=\operatorname{sign}\left(d_{2}\right) \widehat{r}_{2} \pi_{2}
$$

with

$$
\begin{aligned}
\pi_{2}= & -c_{2} \xi_{2}(t)-\widehat{d}_{1} g_{0} \xi_{1}(t)+\frac{\partial \alpha_{1}}{x_{0}(t)} \widehat{d}_{0} g_{0} x_{0}(t) \\
& +\frac{\partial \alpha_{1}}{z_{1}(t)} g_{0}\left[\widehat{d}_{1} z_{2}(t)-\widehat{d}_{0} z_{1}(t)\right]+\frac{\partial \alpha_{1}}{\widehat{\vartheta}_{0}} \dot{\widehat{\vartheta}}_{0} \\
& -\xi_{2}(t) \gamma_{20} \widehat{v}-\xi_{2}(t) \gamma_{21} \widehat{\vartheta}_{1} \\
& -\frac{1}{2 \varepsilon_{21}} \xi_{2}(t)\left(\frac{\partial \alpha_{1}}{z_{1}(t)}\right)^{2} \xi_{1}^{2}(t) e^{2 x_{0}(t)} \\
& -\frac{1}{2 \varepsilon_{22}} \xi_{2}(t) \xi_{1}^{2}(t) x_{0}^{2}(t) \\
& +\frac{\partial \alpha_{1}}{\widehat{r}_{0}} \dot{\widehat{r}}_{0}+\frac{\partial \alpha_{1}}{\widehat{r}_{1}} \dot{\hat{r}}_{1} \\
& +\frac{\partial \alpha_{1}}{\widehat{d}_{0}}\left[-g_{0} \xi_{1}^{2}(t)-\frac{\partial \alpha_{1}}{x_{0}(t)} g_{0} x_{0}(t) \xi_{2}(t)\right. \\
& +\frac{\partial \alpha_{1}}{\widehat{\vartheta}_{1}}\left[\xi_{1}^{2}(t) \widetilde{\omega}_{11}+\xi_{2}^{2}(t) \gamma_{21}\right] \\
z_{1}(t) & \left.g_{0} \xi_{1}(t) \xi_{2}(t)\right]
\end{aligned}
$$

where the virtual control function $\alpha_{1}$ can be expressed as follows:

$$
\begin{aligned}
\alpha_{1}=\operatorname{sign}\left(d_{1}\right) \widehat{r}_{1} \pi_{1}, & \\
\pi_{1}=\widehat{d}_{0} \xi_{1}(t)-\frac{1}{g_{0}} & {\left[c_{1} \xi_{1}(t)+\xi_{1}(t) \widetilde{\omega}_{11} \widehat{\vartheta}_{1}\right.} \\
& \left.+\frac{1}{2 \varepsilon_{11}} \xi_{1}^{3}(t) e^{2 x_{0}(t)}+\frac{\varepsilon_{11}}{8} \xi_{1}^{3}(t) x_{0}^{4}(t)\right] .
\end{aligned}
$$

It can be seen that $\varepsilon_{11}, \varepsilon_{21}$, and $\varepsilon_{22}$ are positive constants; $\gamma_{20}=1$; and $\widetilde{\omega}_{11}$ and $\gamma_{21}$ represent, respectively,

$$
\widetilde{\omega}_{11}=e^{(1 / 8) \ln ^{2}\left(1+x_{0}^{2}(t)\right)}+e^{(1 / 2) z_{1}^{2}(t) x_{0}^{2}(t)},
$$

$$
\begin{aligned}
\gamma_{21}=\frac{1}{2}\left\{\operatorname { s i g n } ( d _ { 1 } ) \widehat { r } _ { 1 } \left[\widehat{d}_{0}-\frac{1}{g_{0}}\left(c_{1}\right.\right.\right. & +\widetilde{\omega}_{11} \widehat{\vartheta}_{1} \\
& +\frac{1}{2 \varepsilon_{11}} \xi_{1}^{2}(t) e^{2 x_{0}(t)} \\
& \left.\left.\left.+\frac{\varepsilon_{11}}{8} \xi_{1}^{2}(t) x_{0}^{4}(t)\right)\right]\right\}^{2} \\
+ & \frac{1}{4}\left(\frac{\partial \alpha_{1}}{x_{0}(t)}\right)^{2} \omega_{0}^{2}+\frac{1}{2}\left(\frac{\partial \alpha_{1}}{z_{1}(t)}\right)^{2} \widetilde{\omega}_{11}^{2} .
\end{aligned}
$$




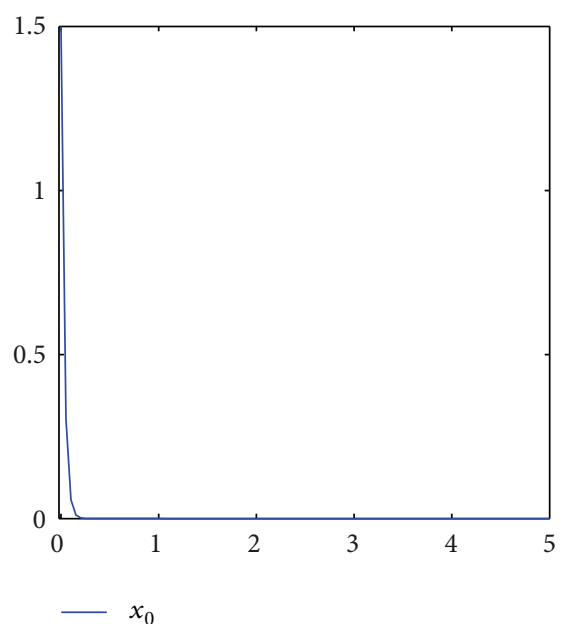

(a)

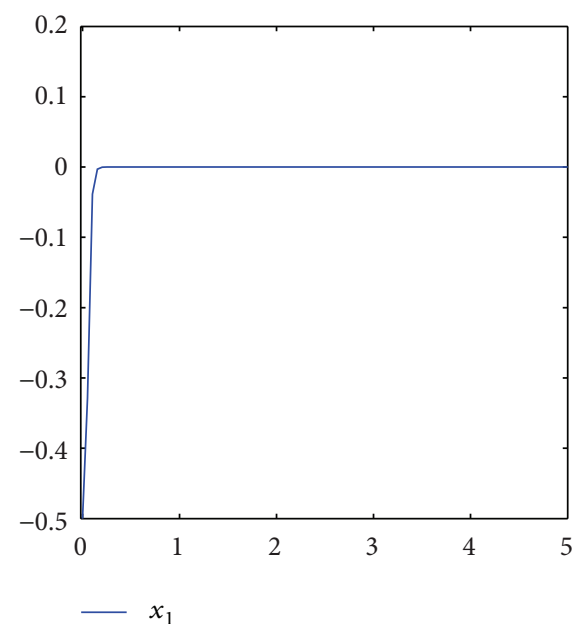

(b)

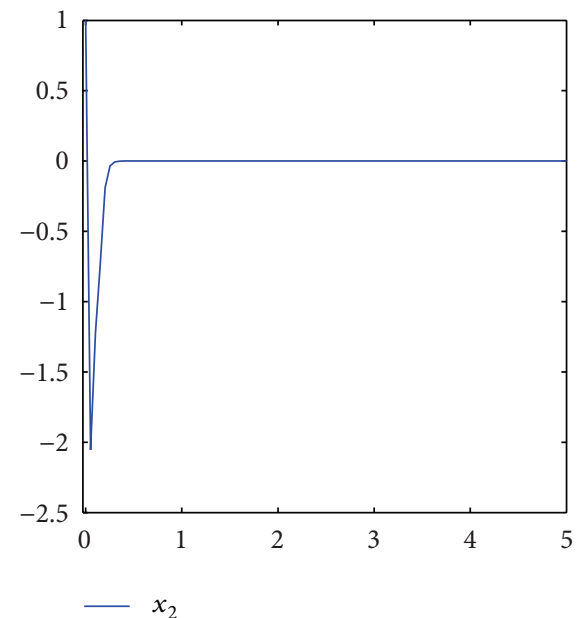

(c)

Figure 1: States $x_{0}(t), x_{1}(t)$, and $x_{2}(t)$.

The adaptation laws of the variables $\widehat{r}_{1}, \widehat{r}_{2}, \widehat{d}_{0}$, and $\widehat{d}_{1}$ in the controller $u_{1}(t)$ and $\widehat{v}, \widehat{\vartheta}_{1}$ are chosen as

$$
\begin{gathered}
\dot{\vec{r}}_{1}=-g_{0} \pi_{1} \xi_{1}(t), \quad \dot{\hat{r}}_{2}=-\pi_{2} \xi_{2}(t), \\
\dot{\vec{d}}_{0}=-g_{0} \xi_{1}^{2}(t)-\frac{\partial \alpha_{1}}{x_{0}(t)} g_{0} x_{0}(t) \xi_{2}(t) \\
+\frac{\partial \alpha_{1}}{z_{1}(t)} g_{0} \xi_{1}(t) \xi_{2}(t), \\
\dot{\vec{d}}_{1}=g_{0} \xi_{1}(t) \xi_{2}(t)-\frac{\partial \alpha_{1}}{z_{1}(t)} g_{0} \xi_{2}(t) z_{2}(t), \\
\dot{\hat{v}}=\xi_{2}^{2}(t) \gamma_{20}, \quad \dot{\hat{\vartheta}}_{1}=\xi_{1}^{2}(t) \widetilde{\omega}_{11}+\xi_{2}^{2}(t) \gamma_{21} .
\end{gathered}
$$

For simulation use, we pick $d_{0}=3.5, d_{1}=2.5, d_{2}=3$, $\theta_{0}=1.5, \theta_{1}=0.5, \theta_{2}=0.5$, and the initial state condition $[1.5-0.51]^{T}$, and the initial values of parameter estimates $\widehat{d}_{0}$, $\widehat{d}_{1}, \widehat{r}_{0}, \widehat{r}_{1}, \widehat{\vartheta}_{0}, \widehat{v}$, and $\widehat{\vartheta}_{1}$ are $0.5,1.5,0.1,0.5,0.3,0.2,0.1$, and 0.5 , respectively (Figures 3,4 , and 5 ). In addition, we take the other parameters as $k_{0}=5, c_{0}=3, c_{1}=2, c_{2}=5, \varepsilon_{11}=2$, and $\varepsilon_{21}=\varepsilon_{22}=1$. Simulation results are shown in Figures 1 and 2 . It is obvious that the states $x_{0}(t), x_{1}(t)$, and $x_{2}(t)$ of the system (62) converge to zero, and the control laws $u_{0}(t)$ and $u_{1}(t)$ also tend to zero.

\section{Conclusion}

The state feedback adaptive stabilization was investigated for a new class of nonholonomic systems with unknown virtual control coefficients, nonlinear uncertainties, and unknown time delays. It should be mentioned that the stabilization approaches in some existing literature resources may fail to be applied for the concerned systems. In order to overcome the difficulties from the time delay, we introduce novel Lyapunov-Krasovskii functionals, and a recursive technique

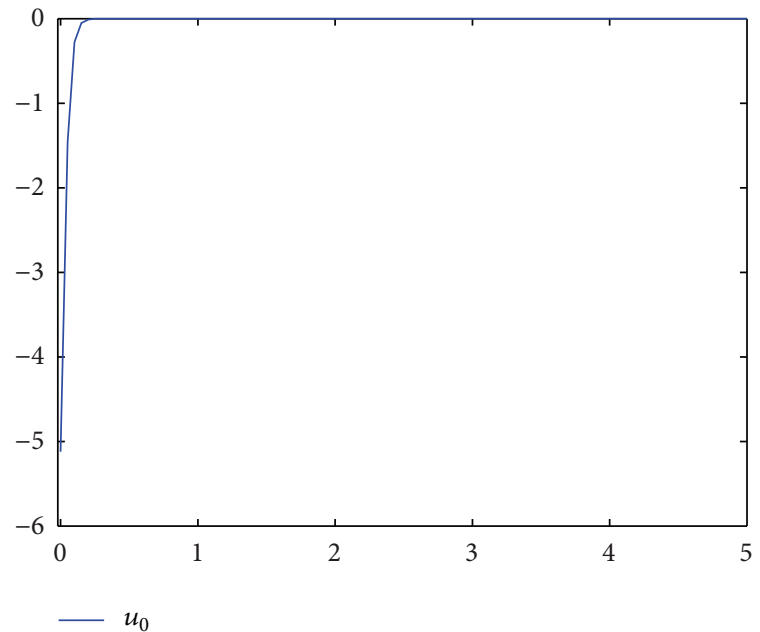

(a)

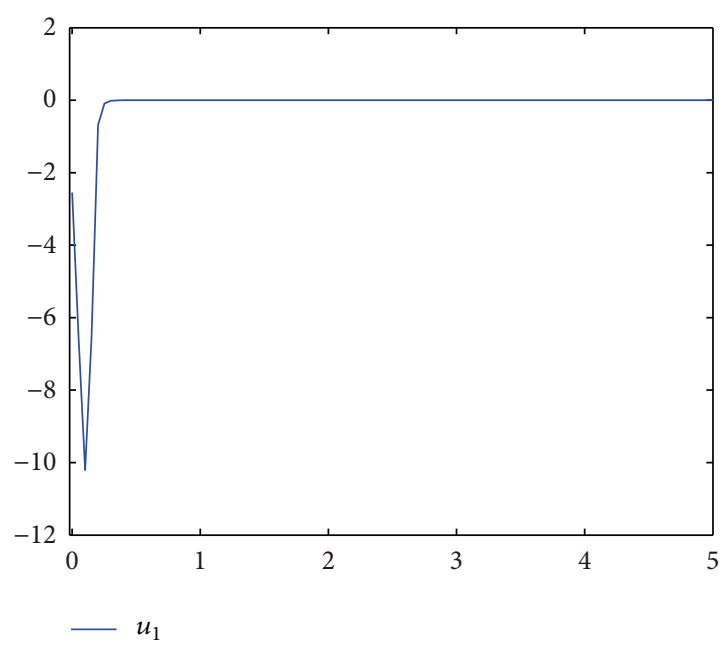

(b)

Figure 2: Controllers $u_{0}(t)$ and $u_{1}(t)$ 


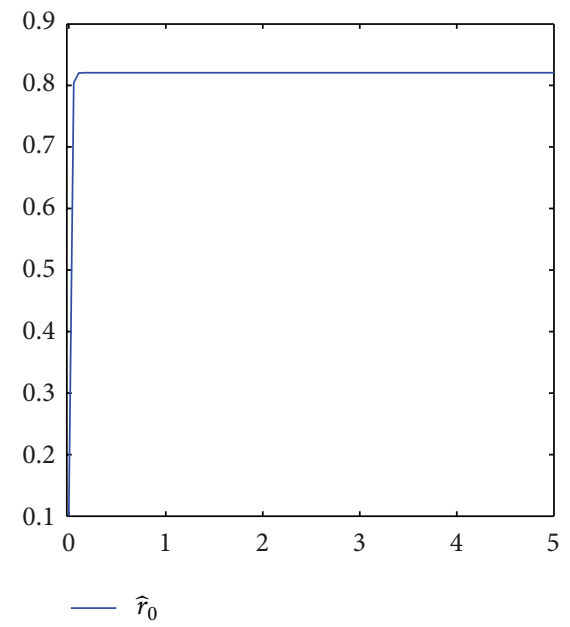

(a)

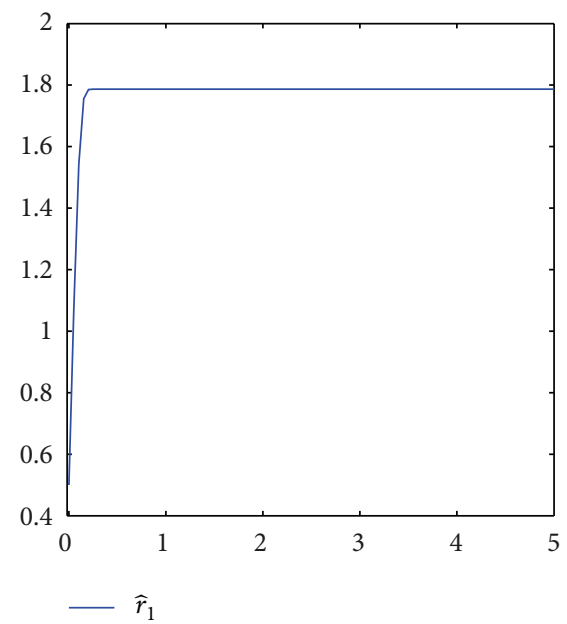

(b)

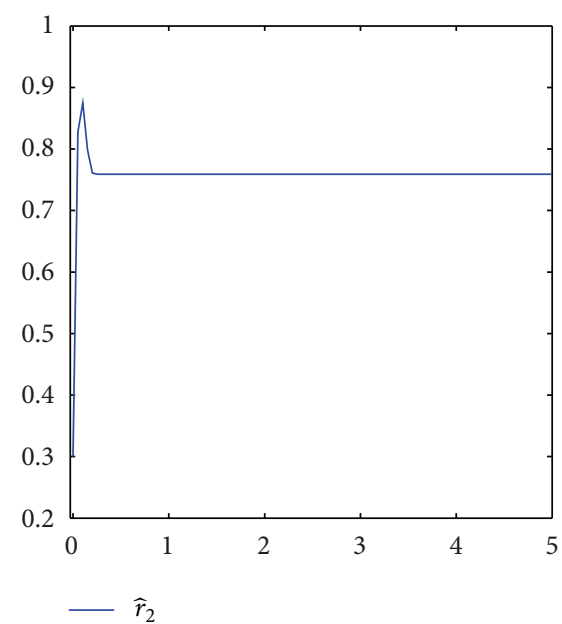

(c)

FIGURE 3: Parameters $\widehat{r}_{0}, \widehat{r}_{1}$, and $\widehat{r}_{2}$.

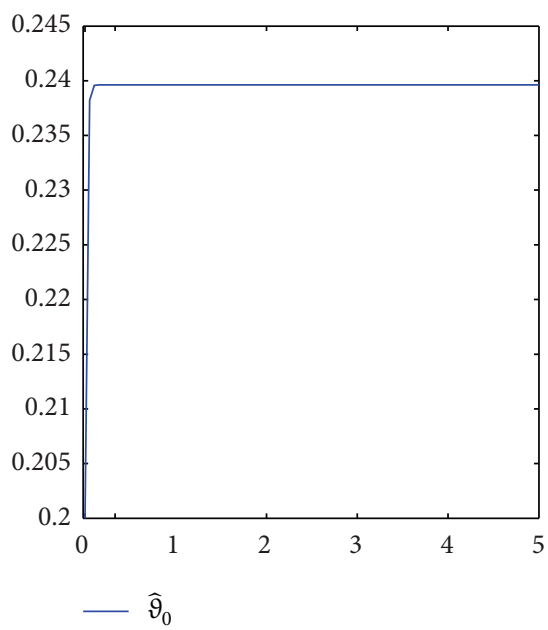

(a)

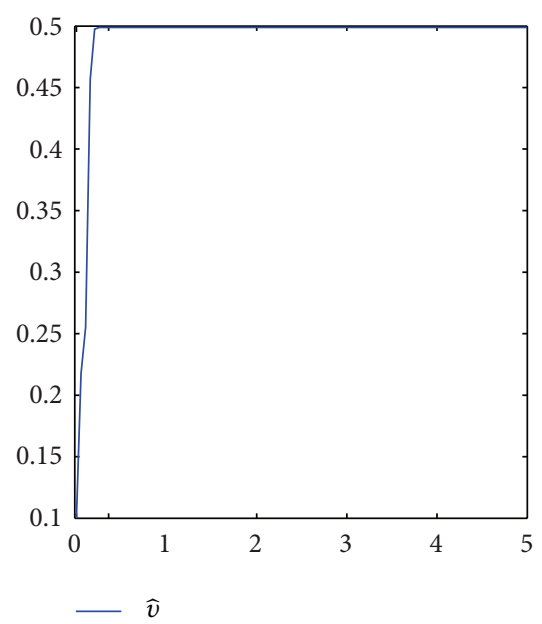

(b)

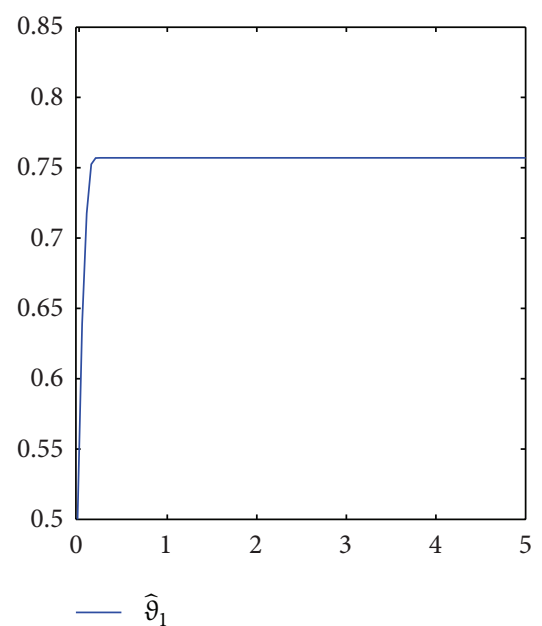

(c)

FIGURE 4: Parameters $\widehat{\vartheta}_{0}, \widehat{v}_{1}$, and $\widehat{\vartheta}_{1}$. 


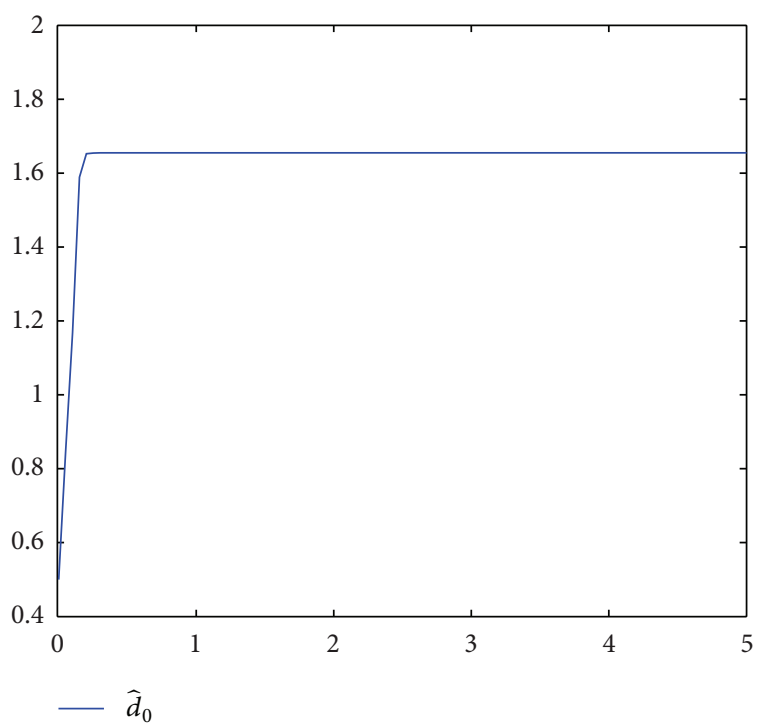

(a)

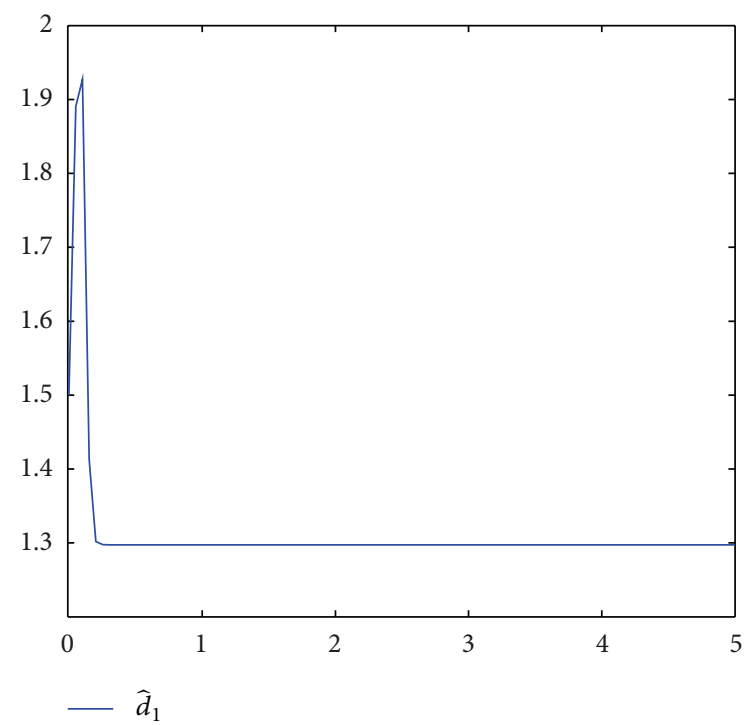

(b)

FIGURE 5: Parameters $\widehat{d}_{0}$ and $\widehat{d}_{1}$.

is proposed to design the adaptive controller. To make the state scaling transformation effective, the switching control strategy is employed to achieve the asymptotic stabilization.

\section{Acknowledgments}

This work is partially supported by Basic and Frontier Technologies Research Program of Henan Province (122300410279) and Doctoral Fund of Zhengzhou University of Light Industry (201BSJJ006).

\section{References}

[1] R. W. Brockett, "Asymptotic stability and feedback stabilization," in Differential Geometric Control Theory, R. W. Brockett, R. S.
Millman, and H. J. Sussmann, Eds., vol. 27 of Progress in Mathematics, pp. 181-191, Birkhäuser, Boston, Mass, USA, 1983.

[2] M. A. Krasnoselskii and P. P. Zabrěko, Geometrical Methods of Nonlinear Analysis, vol. 263 of Fundamental Principles of Mathematical Sciences, Springer, Berlin, Germany, 1983.

[3] A. M. Bloch and N. H. McClamroch, "Control of mechanical systems with classical nonholonomic constraints," in Proceedings of the 28th IEEE Conference on Decision and Control (CDC '89), pp. 201-205, Tampa, Fla, USA, 1989.

[4] A. M. Bloch, M. Reyhanoglu, and N. H. McClamroch, "Control and stabilization of nonholonomic dynamic systems," IEEE Transactions on Automatic Control, vol. 37, no. 11, pp. 1746-1757, 1992.

[5] A. Astolfi, "Discontinuous control of nonholonomic systems," Systems and Control Letters, vol. 27, no. 1, pp. 37-45, 1996.

[6] W. Lin, R. Pongvuthithum, and C. Qian, "Control of high-order nonholonomic systems in power chained form using discontinuous feedback," IEEE Transactions on Automatic Control, vol. 47, no. 1, pp. 108-115, 2002.

[7] J. Luo and P. Tsiotras, "Control design for chained-form systems with bounded inputs," Systems and Control Letters, vol. 39, no. 2, pp. 123-131, 2000.

[8] W. Lin, "Time-varying feedback control of nonaffine nonlinear systems without drift," Systems and Control Letters, vol. 29, no. 2, pp. 101-110, 1996.

[9] R. M'Closkey and R. Murray, "Exponential stabilization of driftless nonlinear control systems using homogeneous feedback," IEEE Transactions on Automatic Control, vol. 42, no. 4, pp. 614628, 1997.

[10] Y.-P. Tian and S. H. Li, "Exponential stabilization of nonholonomic dynamic systems by smooth time-varying control," Automatica, vol. 38, no. 8, pp. 1139-1146, 2002.

[11] Z.-P. Jiang, "Iterative design of time-varying stabilizers for multi-input systems in chained form," Systems and Control Letters, vol. 28, no. 3, pp. 255-262, 1996.

[12] Z.-P. Jiang, "Robust exponential regulation of nonholonomic systems with uncertainties," Automatica, vol. 36, no. 2, pp. 189209, 2000.

[13] O. J. Sørdalen and O. Egeland, "Exponential stabilization of nonholonomic chained systems," IEEE Transactions on Automatic Control, vol. 40, no. 1, pp. 35-49, 1995.

[14] Z.-P. Jiang and H. Nijmeijer, "A recursive technique for tracking control of nonholonomic systems in chained form," IEEE Transactions on Automatic Control, vol. 44, no. 2, pp. 265-279, 1999.

[15] S. S. Ge, Z. P. Wang, and T. H. Lee, "Adaptive stabilization of uncertain nonholonomic systems by state and output feedback," Automatica, vol. 39, no. 8, pp. 1451-1460, 2003.

[16] X. W. Mu, J. M. Yu, and G. F. Cheng, "Adaptive regulation of high order nonholonomic systems," Applied Mathematics and Mechanics, vol. 27, no. 4, pp. 501-507, 2006

[17] F. Z. Gao, F. S. Yuan, and H. J. Yao, "Robust adaptive control for nonholonomic systems with nonlinear parameterization," Nonlinear Analysis: Real World Applications, vol. 11, no. 4, pp. 3242-3250, 2010.

[18] F. Z. Gao, F. S. Yuan, H. J. Yao, and X. W. Mu, "Adaptive stabilization of high order nonholonomic systems with strong nonlinear drifts," Applied Mathematical Modelling, vol. 35, no. 9, pp. 4222-4233, 2011.

[19] Y. Y. Wu and Y. Y. Wu, "Robust stabilization of delayed nonholonomic systems with strong nonlinear drifts," Nonlinear Analysis, vol. 11, no. 5, pp. 3620-3627, 2010. 
[20] Y. Y. Wu and Y. Y. Wu, "Robust stabilization for nonholonomic systems with state delay and nonlinear drifts," Journal of Control Theory and Applications, vol. 9, no. 2, pp. 256-260, 2011.

[21] Z. Xi, G. Feng, Z. P. Jiang, and D. Cheng, "Output feedback exponential stabilization of uncertain chained systems," Journal of the Franklin Institute, vol. 344, no. 1, pp. 36-57, 2007.

[22] Y.-G. Liu and J.-F. Zhang, "Output-feedback adaptive stabilization control design for non-holonomic systems with strong non-linear drifts," International Journal of Control, vol. 78, no. 7, pp. 474-490, 2005.

[23] G. L. Ju, Y. Y. Wu, and W. Sun, "Adaptive output feedback asymptotic stabilization of nonholonomic systems with uncertainties," Nonlinear Analysis. Theory, Methods and Applications, vol. 71, no. 11, pp. 5106-5117, 2009.

[24] X. X. Zheng and Y. Q. Wu, "Adaptive output feedback stabilization for nonholonomic systems with strong nonlinear drifts," Nonlinear Analysis. Theory, Methods and Applications, vol. 70, no. 2, pp. 904-920, 2009.

[25] Y. Y. Wu, Z. H. Diao, and Y. Y. Wu, "Output feedback stabilization of nonholonomic systems with unknown time delays," in Proceedings of the 31st Chinese Control Conference (CCC '12), pp. 625-630, Hefei, China, 2012. 


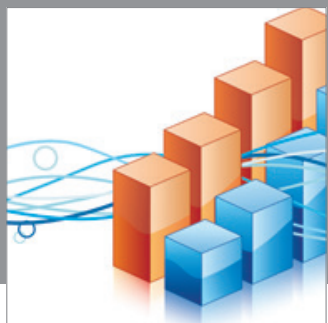

Advances in

Operations Research

mansans

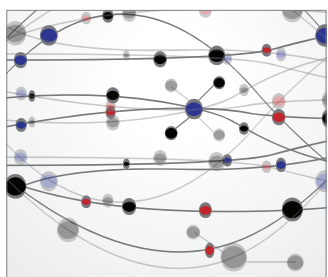

The Scientific World Journal
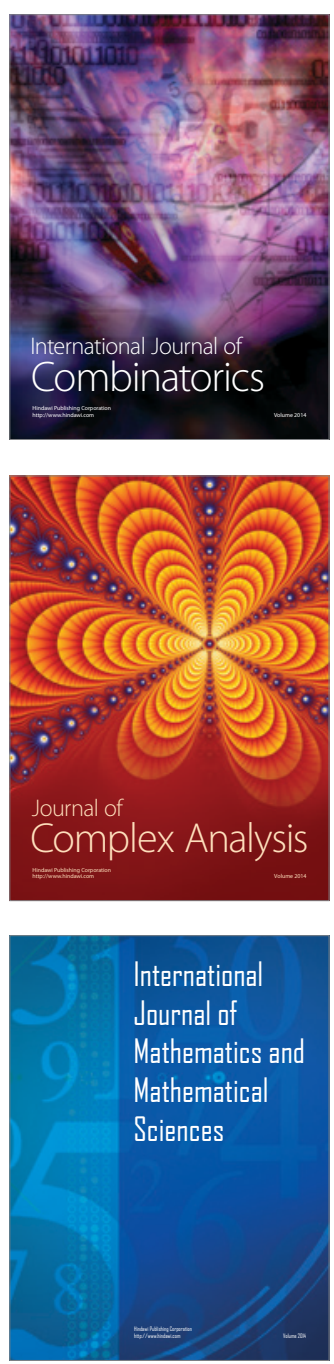
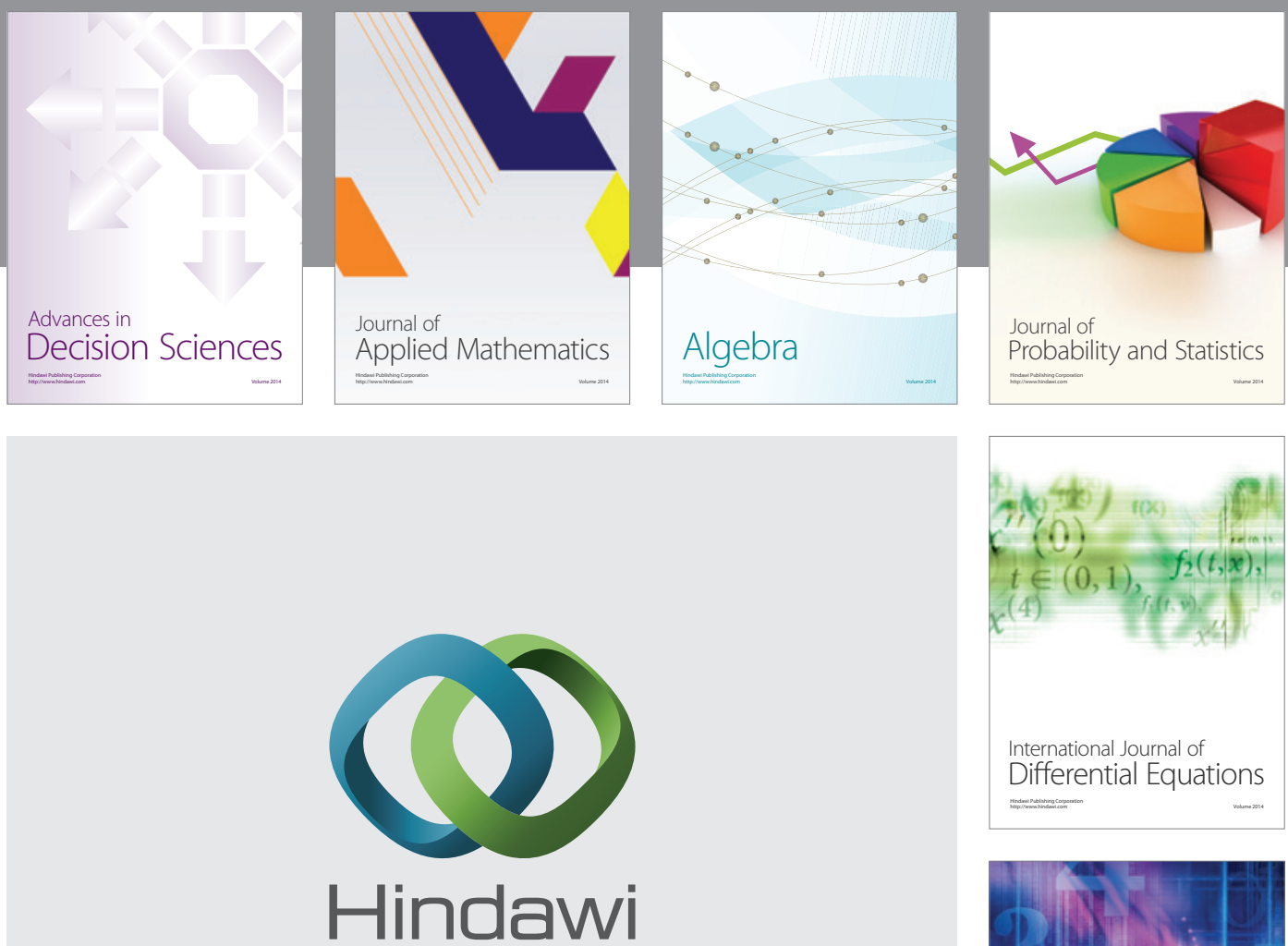

Submit your manuscripts at http://www.hindawi.com
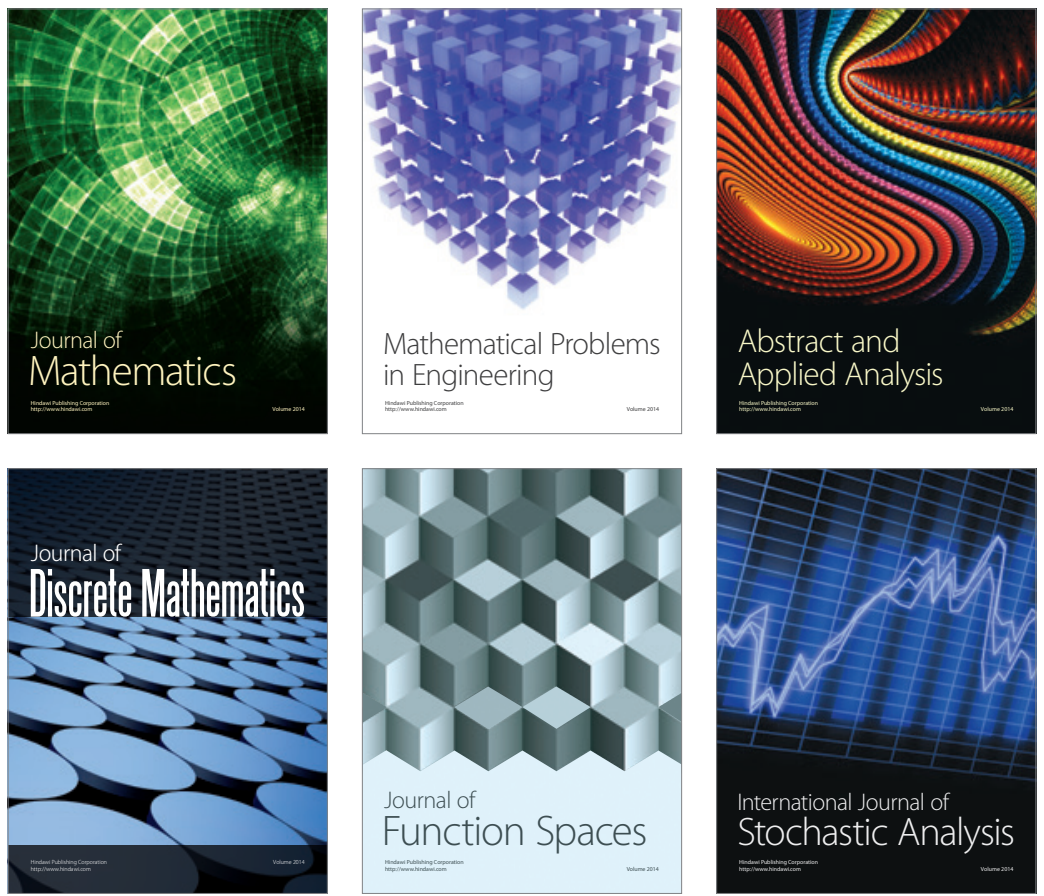

Journal of

Function Spaces

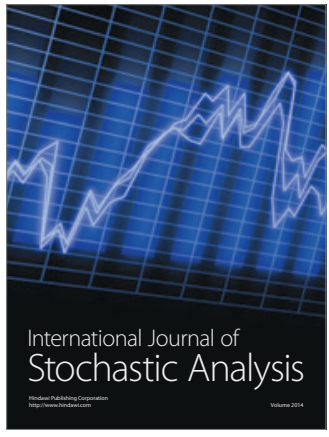

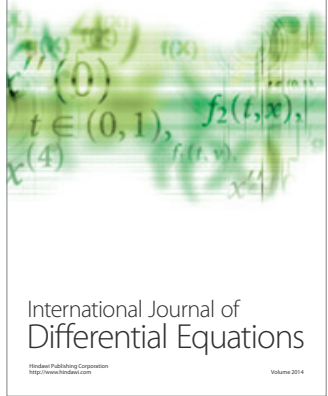
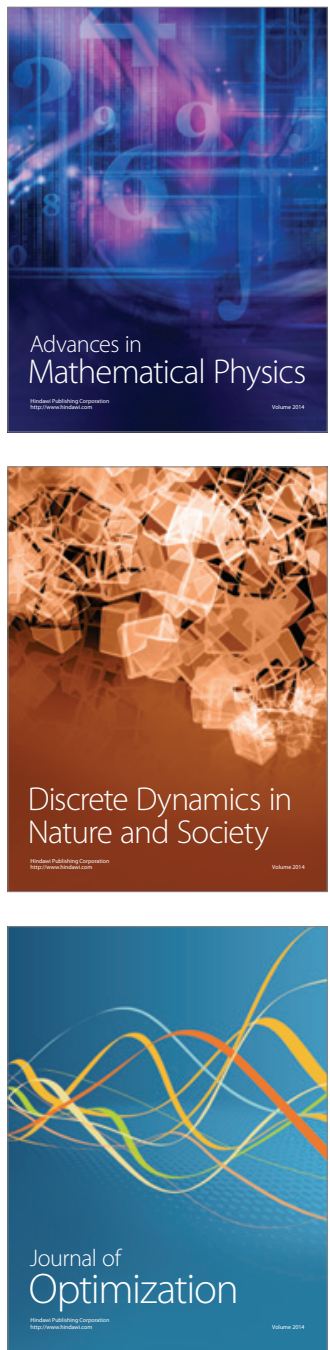